YITP-SB-1015

\title{
Two-loop matching factors for light quark masses and three-loop mass anomalous dimensions in the RI/SMOM schemes
}

\author{
Leandro G. Almeida ${ }^{\mathrm{a}, \mathrm{b}}$ and Christian Sturm ${ }^{\mathrm{b}}$ \\ ${ }^{a}$ C.N. Yang Institute for Theoretical Physics, Stony Brook University, \\ Stony Brook, New York 11794, USA \\ b Physics Department, Brookhaven National Laboratory, \\ Upton, New York 11973, USA
}

\begin{abstract}
Light quark masses can be determined through lattice simulations in regularization invariant momentum-subtraction(RI/MOM) schemes. Subsequently, matching factors, computed in continuum perturbation theory, are used in order to convert these quark masses from a RI/MOM scheme to the $\overline{\mathrm{MS}}$ scheme. We calculate the two-loop corrections in quantum chromodynamics(QCD) to these matching factors as well as the three-loop mass anomalous dimensions for the RI/SMOM and RI/SMOM $\gamma_{\mu}$ schemes. These two schemes are characterized by a symmetric subtraction point. Providing the conversion factors in the two different schemes allows for a better understanding of the systematic uncertainties. The two-loop expansion coefficients of the matching factors for both schemes turn out to be small compared to the traditional RI/MOM schemes. For $n_{f}=3$ quark flavors they are about $0.6 \%-0.7 \%$ and $2 \%$, respectively, of the leading order result at scales of about $2 \mathrm{GeV}$. Therefore, they will allow for a significant reduction of the systematic uncertainty of light quark mass determinations obtained through this approach. The determination of these matching factors requires the computation of amputated Green's functions with the insertions of quark bilinear operators. As a by-product of our calculation we also provide the corresponding results for the tensor operator.
\end{abstract}




\section{Introduction}

Light quark masses, like the up-, down-, and strange-quark masses, are fundamental parameters of the Standard Model, and their precise determination is thus an impor-

tant task. They can be determined, for example, with the help of lattice simulations in combination with nonperturbative renormalization(NPR). In this context regularization invariant momentum-subtraction (RI/MOM) schemes [1] play a crucial role; for a recent overview see e.g. Ref. [2].

In Ref. [3] these light quark masses were determined in the RI/MOM scheme and subsequently converted to the $\overline{\mathrm{MS}}$ scheme [4,5]. This conversion requires the computation of a matching factor $C_{m}^{x}$, which performs this transformation of the quark mass from the scheme $x$ into the $\overline{\mathrm{MS}}$ mass. Since the RI/MOM schemes do not depend on the particular regulator which has been used to regularize the ultraviolet divergences, this matching factor can be calculated in continuum perturbation theory using dimensional regularization [6]. In the $\mathrm{RI} / \mathrm{MOM}$ scheme this matching factor is known up to three-loop order in perturbative QCD [1,7,8]; the same holds for the $\mathrm{RI}^{\prime} / \mathrm{MOM}$ [8, 9] scheme.

Both the RI/MOM and $\mathrm{RI}^{\prime} / \mathrm{MOM}$ schemes employ a renormalization procedure which uses an exceptional subtraction point. Here the subtraction point defines the configuration of the external momenta of the considered amplitude, where the ultraviolet divergences are subtracted. In continuum perturbation theory, mass and fermion field renormalization constants are typically computed by considering higher order corrections to fermion selfenergy diagrams. The fermion self-energies are related through Ward-Takahashi identities to amputated Green's functions of quark bilinear operators, i.e. the vector, axial-vector, scalar and pseudoscalar operators. These relations allow an extraction of the renormalization constants from these amputated Green's functions with operator insertions, rather than from self-energies. In the case of an exceptional momentum configuration, no momentum transfer leaves the operator.

However, at low scales $(\sim 2 \mathrm{GeV})$ the perturbative expansion of the matching factors for the RI/MOM and RI'/MOM schemes exhibit a poor convergence behavior and, as a result, introduce large systematic uncertainties in the determination of the $\overline{\mathrm{MS}}$ masses of the light quark sector. This amounts to approximately $60 \%$ of the total error. Apart from that, the lattice simulations are, for an exceptional subtraction point, more prone to unwanted infrared effects. For this reason, the use of a symmetric subtraction point was proposed in Ref. [10], and the concepts and framework of these new RI/SMOM schemes have been worked out in Ref. [11]. A nonexceptional or symmetric subtraction point is characterized by the fact that a momentum leaves the operator of the amputated Green's function, and in the case of a symmetric subtraction point, the squares of all momenta leaving the amplitude are equal. A nonperturbative test of such a RI/SMOM scheme can be found in Ref. [12. The one-loop QCD corrections of these matching factors in the RI/SMOM schemes are known [11], and are shown to have a better convergence behavior than the traditional RI/MOM and $\mathrm{RI}^{\prime} / \mathrm{MOM}$ schemes. However, the question of whether this behavior persists at higher orders in perturbation theory still remained unanswered. If it were confirmed, this would lead to a significant reduction of the systematic uncertainties 
associated with the determination of the light quark masses.

The goal of this paper is to extend the work of Ref. [11] and to provide the two-loop QCD corrections to the matching factors of the so-called RI/SMOM and RI/SMOM $\gamma_{\mu}$ schemes as well as the three-loop mass anomalous dimensions.

This paper is structured as follows: In Section 2 we introduce the notations and conventions that are used. In Section 3 we give an outline of the perturbative calculation, and in Section 4 we present the results for the matching factors and the mass anomalous dimensions. Finally, in Section 5 we close with a summary and conclusions. In the appendixes we provide additional information about some master integrals and, for completeness, results for conversion factors with complete gauge parameter dependence as well as results for anomalous dimensions.

\section{Generalities and Notation}

In Ref. [1] two regularization invariant momentum-subtraction schemes with a symmetric subtraction point have been defined, the so-called RI/SMOM and RI/SMOM $\gamma_{\mu}$ schemes. The RI/SMOM(RI/SMOM $\left.\gamma_{\mu}\right)$ scheme can be seen as an extension of the $\mathrm{RI}^{\prime} / \mathrm{MOM}(\mathrm{RI} / \mathrm{MOM})$ scheme from an exceptional to a nonexceptional subtraction point. The renormalization conditions of these two new schemes are given in Eqs. (10), (11), and (A15) of Ref. [11] and allow for a determination of the renormalization constants of the fermion field $\Psi$ and the quark mass $m$ through the computation of the nonsinglet amputated Green's function $\Lambda_{\hat{O}}$ with the insertion of quark bilinear operators $\hat{O}$, that is to say, the scalar $(\hat{O}=S)$, the pseudoscalar $(\hat{O}=P)$, the vector $(\hat{O}=V)$, and the axial-vector $(\hat{O}=A)$ operators. Further details can be found in Ref. [11], whose conventions we follow for these quantities.

In the determination of the light quark mass through lattice simulations, the RI/SMOM and RI/SMOM $\gamma_{\mu}$ schemes serve as intermediate schemes before the conversion of the quark mass to the $\overline{\mathrm{MS}}$ scheme. This conversion is performed in continuum perturbation theory through the computation of a matching factor $C_{m}^{x}$ with the property

$$
m_{R}^{\overline{\mathrm{MS}}}=C_{m}^{x} m_{R}^{x}, \quad x \in\left\{\mathrm{RI} / \mathrm{SMOM}, \mathrm{RI} / \mathrm{SMOM}_{\gamma_{\mu}}\right\} .
$$

The index $R$ denotes here and in the following a renormalized quantity. As shown in Ref. [1] these conversion factors can be extracted from the amputated Green's function of the pseudoscalar(or scalar) operator through the equation

$$
\left(C_{m}^{x}\right)^{-1}=\left.C_{q}^{x} \lim _{m_{R} \rightarrow 0} \frac{1}{12 i} \operatorname{Tr}\left[\Lambda_{P, R}^{\overline{\mathrm{MS}}}\left(p_{1}, p_{2}\right) \gamma_{5}\right]\right|_{\text {sym }}, \quad x \in\left\{\mathrm{RI} / \mathrm{SMOM}, \mathrm{RI} / \mathrm{SMOM}_{\gamma_{\mu}}\right\},
$$

where $C_{q}^{x}$ is the corresponding matching factor for the fermion field which converts the field from the RI/SMOM(RI/SMOM $\left.\gamma_{\mu}\right)$ scheme to the $\overline{\mathrm{MS}}$ scheme via the equation

$$
\Psi_{R}^{\overline{\mathrm{MS}}}=\sqrt{C_{q}^{x}} \Psi_{R}^{x}, \quad x \in\left\{\mathrm{RI} / \mathrm{SMOM}, \mathrm{RI} / \mathrm{SMOM}_{\gamma_{\mu}}\right\} .
$$


The matching factor $C_{q}^{x}$ can be determined through the computation of the amputated Green's function with the insertion of the axial-vector(or vector) operator

$$
\left(C_{q}^{\mathrm{RI} / \mathrm{SMOM}}\right)^{-1}=\left.\lim _{m_{R} \rightarrow 0} \frac{1}{12 q^{2}} \operatorname{Tr}\left[q_{\mu} \Lambda_{A, R}^{\mu, \overline{\mathrm{MS}}}\left(p_{1}, p_{2}\right) \gamma_{5} \not q\right]\right|_{\text {sym }}
$$

and

$$
\left(C_{q}^{\mathrm{RI} / \mathrm{SMOM}_{\gamma_{\mu}}}\right)^{-1}=\left.\lim _{m_{R} \rightarrow 0} \frac{1}{48} \operatorname{Tr}\left[\Lambda_{A, R}^{\mu, \overline{\mathrm{MS}}}\left(p_{1}, p_{2}\right) \gamma_{5} \gamma_{\mu}\right]\right|_{s y m},
$$

where Eqs. (41) and (5) are distinguished through the use of two different projectors in the trace with which the amputated Green's functions are multiplied. The subscript sym in Eqs. (2), (41) and (5) stands for the restriction of the amputated Green's function to the symmetric momentum configuration

$$
\left.f\left(p_{1}^{2}, p_{2}^{2}, q^{2}\right)\right|_{\text {sym }}=\left.f\left(p_{1}^{2}, p_{2}^{2}, q^{2}\right)\right|_{p_{1}^{2}=p_{2}^{2}=q^{2}=-\mu^{2}},
$$

where $p_{1}$ and $p_{2}$ are the momenta of the external fermions and $q=p_{1}-p_{2}$ is the momentum transfer leaving the operator. The momenta are also defined in the two-loop diagrams of Fig. 1. The symbol $\mu^{2}>0$ denotes the renormalization scale, which we choose for the $\mathrm{RI} / \mathrm{SMOM}\left(\mathrm{RI} / \mathrm{SMOM}_{\gamma_{\mu}}\right)$ scheme to be equal to the one in the $\overline{\mathrm{MS}}$ scheme.

Also, the tensor operator $(\hat{O}=T)$ has interesting applications in the context of lattice simulations; see e.g. Ref. [13]. Following the notation and Eq. (12) of Ref. [11] one obtains the corresponding matching factor $C_{T}^{x}$, which converts the tensor operator from the $x$ scheme to the $\overline{\mathrm{MS}}$ scheme,

$$
\hat{O}_{R}^{\overline{\mathrm{MS}}}=C_{\hat{O}}^{x} \hat{O}_{R}^{x}
$$

by computing

$$
C_{T}^{x}=\left.C_{q}^{x} \lim _{m_{R} \rightarrow 0} \frac{1}{144} \operatorname{Tr}\left[\Lambda_{T, R}^{\mu \nu, \overline{\mathrm{MS}}}\left(p_{1}, p_{2}\right) \sigma_{\mu \nu}\right]\right|_{\text {sym }}, \quad x \in\left\{\text { RI/SMOM, RI/SMOM } \gamma_{\mu}\right\},
$$

with $\sigma_{\mu \nu}=\frac{i}{2}\left[\gamma_{\mu}, \gamma_{\nu}\right]$.

The conversion factors in Eqs. (1), (3), and (17) are gauge dependent. In the case of the mass conversion factor this gauge dependence compensates for the one in the mass renormalization constant which is obtained through lattice simulations; the NPR procedure of Ref. [1] is gauge dependent. The lattice simulations are typically performed in the Landau gauge $(\xi=0)$. For generality we keep the complete dependence on the gauge parameter and use for the gluon propagator

$$
\frac{i \delta^{a b}}{q^{2}+i \epsilon}\left(-g^{\mu \nu}+(1-\xi) \frac{q^{\mu} q^{\nu}}{q^{2}+i \epsilon}\right) \text {. }
$$

\section{Calculation}

The calculation of the two-loop QCD corrections proceeds in two steps. In the first step, after the generation of the diagrams, all loop integrals that appear are mapped on a small set of master integrals. In the second step these master integrals need to be solved. 

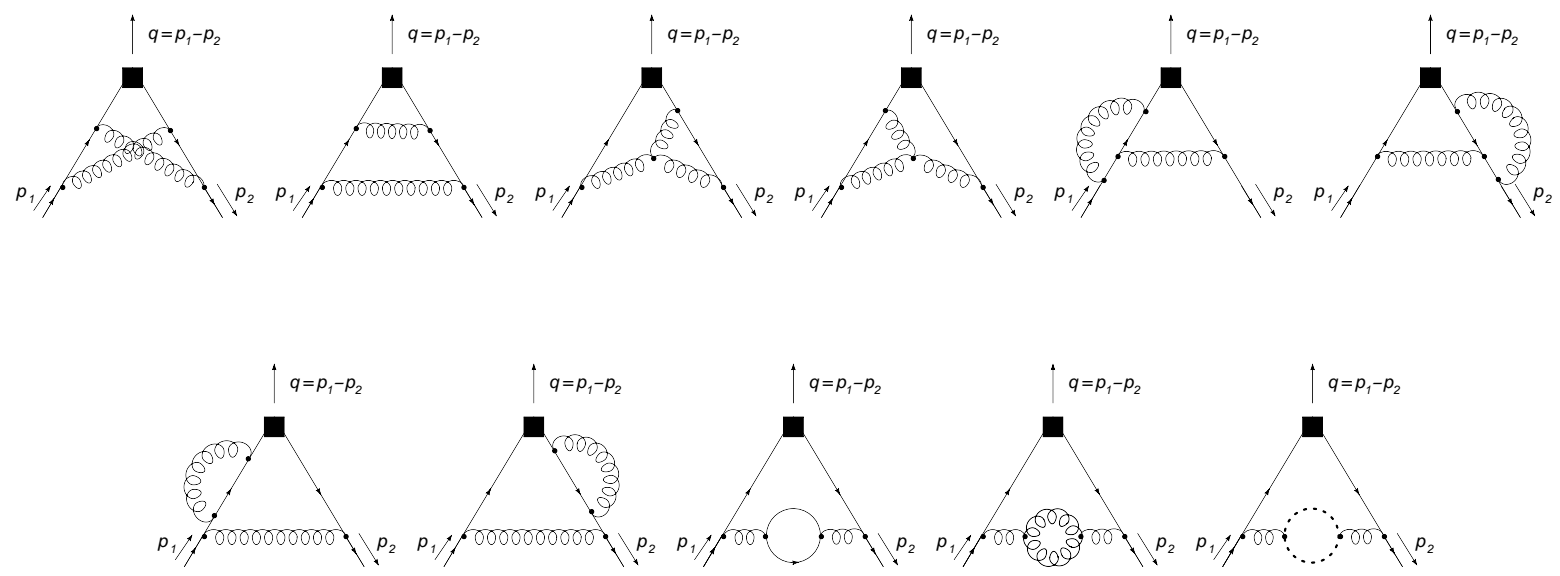

Figure 1: Two-loop Feynman diagrams which contribute to the computation of the nonsinglet, amputated Green's functions. The spiral lines denote gluons, the solid lines represent fermions, the dashed lines are ghost fields, and the black boxes indicate the inserted operator.

\subsection{Manipulation of loop integrals}

In order to generate the required Feynman diagrams, shown in Fig. 1, we have used the program QGRAF [14]. We identify the different topologies and adopt the proper notation with the help of the packages q2e and exp [15 17]. This allows us to prepare an output which can straightforwardly be used to perform the reduction to master integrals. The reduction is achieved with the traditional integration-by-parts (IBP) method [18] in combination with Laporta's algorithm [19, 20] and has been carried out with a FORM [21, 23] based implementation. The rational functions in the space-time dimension $d$, which appear while solving the arising linear system of equations, have been simplified with the program FERMAT [24].

In the case of the computation of the amputated Green's function with the insertion of the pseudoscalar or axial-vector operator, we use a naive anticommuting definition of $\gamma_{5}$ for the treatment of $\gamma_{5}$ in dimensional regularization [6, 25], which is a self-consistent prescription for the flavor nonsinglet contributions considered here [26, 27].

\subsection{Master integrals}

After the IBP reduction of the two-loop amplitude, seven massless master integrals survive. The one- and two-loop master integrals are shown in Fig. 2. They are defined in Minkowskian space in $d=4-2 \varepsilon$ space-time dimensions by

$$
\mathcal{M}_{21}^{(1)}=\mu^{2 \varepsilon} e^{\varepsilon \gamma_{E}} \int \frac{d^{d} \ell_{1}}{i \pi^{d / 2}} \frac{1}{D_{1} D_{2}}, \quad \mathcal{M}_{31}^{(1)}=\mu^{2 \varepsilon} e^{\varepsilon \gamma_{E}} \int \frac{d^{d} \ell_{1}}{i \pi^{d / 2}} \frac{1}{D_{1} D_{2} D_{3}},
$$




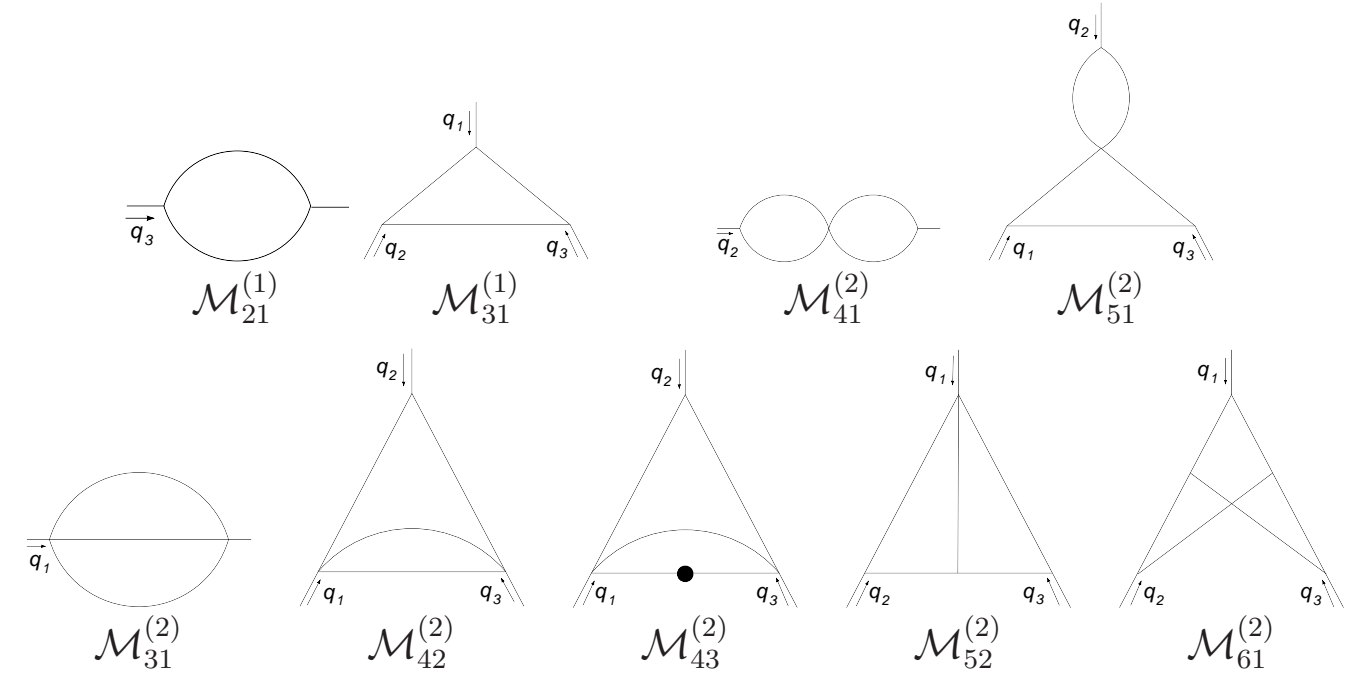

Figure 2: There appear two one-loop master integrals and seven two-loop master integrals from which two are factorized. The symbol $\mathcal{M}_{p r}^{(l)}$ denotes a $l$-loop topology with $p$ lines. The number $r$ serves as a running number to enumerate topologies with the same number of loops and propagators.

$$
\begin{array}{rlrl}
\mathcal{M}_{31}^{(2)} & =\mu^{4 \varepsilon} e^{2 \varepsilon \gamma_{E}} \int \frac{d^{d} \ell_{1}}{i \pi^{d / 2}} \frac{d^{d} \ell_{2}}{i \pi^{d / 2}} \frac{1}{D_{1} D_{4} D_{7}}, & \mathcal{M}_{41}^{(2)} & =\mu^{4 \varepsilon} e^{2 \varepsilon \gamma_{E}} \int \frac{d^{d} \ell_{1}}{i \pi^{d / 2}} \frac{d^{d} \ell_{2}}{i \pi^{d / 2}} \frac{1}{D_{1} D_{3} D_{4} D_{5}}, \\
\mathcal{M}_{42}^{(2)} & =\mu^{4 \varepsilon} e^{2 \varepsilon \gamma_{E}} \int \frac{d^{d} \ell_{1}}{i \pi^{d / 2}} \frac{d^{d} \ell_{2}}{i \pi^{d / 2}} \frac{1}{D_{1} D_{4} D_{5} D_{7}}, & \mathcal{M}_{43}^{(2)} & =\mu^{4 \varepsilon} e^{2 \varepsilon \gamma_{E}} \int \frac{d^{d} \ell_{1}}{i \pi^{d / 2}} \frac{d^{d} \ell_{2}}{i \pi^{d / 2}} \frac{1}{D_{1}^{2} D_{4} D_{5} D_{7}}, \\
\mathcal{M}_{51}^{(2)} & =\mu^{4 \varepsilon} e^{2 \varepsilon \gamma_{E}} \int \frac{d^{d} \ell_{1}}{i \pi^{d / 2}} \frac{d^{d} \ell_{2}}{i \pi^{d / 2}} \frac{1}{D_{1} D_{2} D_{3} D_{4} D_{5}}, & \mathcal{M}_{52}^{(2)} & =\mu^{4 \varepsilon} e^{2 \varepsilon \gamma_{E}} \int \frac{d^{d} \ell_{1}}{i \pi^{d / 2}} \frac{d^{d} \ell_{2}}{i \pi^{d / 2}} \frac{1}{D_{1} D_{2} D_{4} D_{5} D_{6}}, \\
\mathcal{M}_{61}^{(2)} & =\mu^{4 \varepsilon} e^{2 \varepsilon \gamma_{E}} \int \frac{d^{d} \ell_{1}}{i \pi^{d / 2}} \frac{d^{d} \ell_{2}}{i \pi^{d / 2}} \frac{1}{D_{1} D_{2} D_{4} D_{5} D_{6} D_{7}},
\end{array}
$$

with the denominators

$$
\begin{array}{lll}
D_{1}=\ell_{1}^{2}+i \epsilon, & D_{2}=\left(\ell_{1}+q_{3}\right)^{2}+i \epsilon, & D_{3}=\left(\ell_{1}-q_{2}\right)^{2}+i \epsilon, \\
D_{4}=\ell_{2}^{2}+i \epsilon, & D_{5}=\left(\ell_{2}-q_{2}\right)^{2}+i \epsilon, & D_{6}=\left(\ell_{1}-\ell_{2}\right)^{2}+i \epsilon, \\
D_{7}=\left(\ell_{1}-\ell_{2}-q_{1}\right)^{2}+i \epsilon, & &
\end{array}
$$

where $\ell_{1}$ and $\ell_{2}$ are loop momenta and $q_{1}, q_{2}$, and $q_{3}$ are external momenta with $q_{1}^{2}=$ $q_{2}^{2}=q_{3}^{2}=-\mu^{2}$. The symbol $e \simeq 2.71828$ is Euler's number and $\gamma_{E} \simeq 0.577216$ is the Euler-Mascheroni constant.

The one- and two-loop self-energy integrals $\mathcal{M}_{21}^{(1)}, \mathcal{M}_{31}^{(2)}$ are well known to all orders in $\varepsilon$. The factorized master integrals $\mathcal{M}_{41}^{(2)}$ and $\mathcal{M}_{51}^{(2)}$ can be obtained from taking the product of the two one-loop integrals. Each of the master integrals $\mathcal{M}_{42}^{(2)}$ and $\mathcal{M}_{43}^{(2)}$ can be written as a product of a scalar one-loop two-point function and a one-loop threepoint function $\mathcal{M}_{31}^{(1)}$, which has a noninteger power of one of the propagators. The latter has been determined analytically in terms of hypergeometric functions to all orders in 
$\varepsilon$ in Ref. [28]. Algorithms to expand generalized hypergeometric functions in a small parameter, like $\varepsilon$, have been developed in Refs. [29,30]. For the practical implementation we use the Mathematica packages HypExp and HPL 31 33. Expansions of hypergeometric functions can also be found in Refs. [34, 35]. The master integrals $\mathcal{M}_{52}^{(2)}$ and $\mathcal{M}_{61}^{(2)}$ can be taken from Ref. [36] in the special case of symmetric external momenta $q_{i}^{2}=-\mu^{2}$ for $i=1,2,3$. Results for the master integrals in terms of harmonic polylogarithms can also be found in Ref. [37].

The results expanded in $\varepsilon$ to the maximally required order read

$$
\begin{aligned}
\mathcal{M}_{21}^{(1)}= & \frac{1}{\varepsilon}+2+\varepsilon\left[4-\frac{\pi^{2}}{12}\right]+\varepsilon^{2}\left[8-\frac{\pi^{2}}{6}-\frac{7}{3} \zeta_{3}\right]+\mathcal{O}\left(\varepsilon^{3}\right) \\
\mu^{2} \mathcal{M}_{31}^{(1)}= & \left(\frac{2}{3} \pi\right)^{2}-\frac{2}{3} \Psi^{\prime}\left(\frac{1}{3}\right)+\varepsilon\left[12 s_{3}\left(\frac{\pi}{6}\right)-\frac{35}{108} \frac{\pi^{3}}{\sqrt{3}}-\frac{\log ^{2}(3) \pi}{4 \sqrt{3}}\right]+\varepsilon^{2} \mathcal{H}_{31}^{(2)}+\mathcal{O}\left(\varepsilon^{3}\right),(11) \\
\mathcal{M}_{41}^{(2)}= & \frac{1}{\varepsilon^{2}}+\frac{4}{\varepsilon}+12-\frac{\pi^{2}}{6}+\varepsilon\left[32-\frac{2}{3} \pi^{2}-\frac{14}{3} \zeta_{3}\right]+\mathcal{O}\left(\varepsilon^{2}\right) \\
\mu^{2} \mathcal{M}_{51}^{(2)}= & \frac{1}{\varepsilon}\left[\left(\frac{2}{3} \pi\right)^{2}-\frac{2}{3} \Psi^{\prime}\left(\frac{1}{3}\right)\right]+12 s_{3}\left(\frac{\pi}{6}\right)-\frac{35}{108} \frac{\pi^{3}}{\sqrt{3}}-\frac{\log ^{2}(3) \pi}{4 \sqrt{3}}-\frac{4}{3} \Psi^{\prime}\left(\frac{1}{3}\right) \\
& +\frac{8}{9} \pi^{2}+\varepsilon\left[24 s_{3}\left(\frac{\pi}{6}\right)-\frac{35 \pi^{3}}{54 \sqrt{3}}-\frac{\log ^{2}(3) \pi}{2 \sqrt{3}}-\frac{8}{3} \Psi^{\prime}\left(\frac{1}{3}\right)+\frac{16}{9} \pi^{2}+\frac{\pi^{2}}{18} \Psi^{\prime}\left(\frac{1}{3}\right)\right. \\
& \left.-\frac{\pi^{4}}{27}+\mathcal{H}_{31}^{(2)}\right]+\mathcal{O}\left(\varepsilon^{2}\right) \\
\frac{1}{\mu^{2}} \mathcal{M}_{31}^{(2)}= & \frac{1}{4 \varepsilon}+\frac{13}{8}+\varepsilon\left[\frac{115}{16}-\frac{\pi^{2}}{24}\right]+\varepsilon^{2}\left[\frac{865}{32}-\frac{13}{48} \pi^{2}-\frac{8}{3} \zeta_{3}\right]+\mathcal{O}\left(\varepsilon^{3}\right) \\
\mathcal{M}_{42}^{(2)}= & \frac{1}{2 \varepsilon^{2}}+\frac{5}{2 \varepsilon}+\frac{19}{2}-\frac{1}{3} \Psi^{\prime}\left(\frac{1}{3}\right)+\frac{5}{36} \pi^{2}+\varepsilon\left[\frac{65}{2}-12 s_{2}\left(\frac{\pi}{2}\right)+6 s_{2}\left(\frac{\pi}{6}\right)\right. \\
& +8 s_{3}\left(\frac{\pi}{2}\right)-4 s_{3}\left(\frac{\pi}{6}\right)-\frac{67}{324 \sqrt{3}} \pi^{3}-\frac{\pi \log (3)}{2 \sqrt{3}}-\frac{\log ^{2}(3) \pi}{12 \sqrt{3}}-\frac{2}{3} \Psi^{\prime}\left(\frac{1}{3}\right) \\
& \left.+\frac{\pi^{2}}{36}-\frac{10}{3} \zeta_{3}\right]+\mathcal{O}\left(\varepsilon^{2}\right) \\
\mu^{2} \mathcal{M}_{61}^{(2)}= & {\left[\left(\frac{2}{3} \pi\right)^{2}-\frac{2}{3} \Psi^{\prime}\left(\frac{1}{3}\right)\right]^{2}+\mathcal{O}(\varepsilon) } \\
\mu_{52}^{2} \mathcal{M}^{(2)}= & \left.\frac{2}{27} \pi^{4}-\frac{1}{36} \Psi^{\prime}\left(\frac{1}{3}\right)-\left(\frac{2}{3} \pi\right)^{2}\right)+\frac{\pi \log ^{2}(3)}{4 \sqrt{3}}+\frac{35 \pi^{3}}{108 \sqrt{3}}-12 s_{3}\left(\frac{\pi}{6}\right) \\
\mu^{2} \mathcal{M}_{43}^{(2)}= & {\left[\frac{\pi^{4}}{27}-\frac{\pi^{2}}{18} \Psi^{\prime}\left(\frac{1}{3}\right)+\mathcal{H}_{43}^{(2)}\right]+\mathcal{O}\left(\varepsilon^{2}\right) } \\
= & \\
= &
\end{aligned}
$$

where $\Psi(x)$ is the digamma function $\Psi(x)=\Gamma^{\prime}(x) / \Gamma(x)$; here the prime denotes the 
derivative of the $\Gamma$ function. We also define $s_{n}(x)=\frac{1}{\sqrt{3}} \operatorname{Im}\left[\operatorname{Li}_{n}\left(\frac{e^{i x}}{\sqrt{3}}\right)\right]$ with the polylogarithm function $\operatorname{Li}_{n}(z)=\sum_{k=1}^{\infty} \frac{z^{k}}{k^{n}}$. The symbol $\zeta_{n}=\operatorname{Li}_{n}(1)$ is the Riemann zeta function. The constants $\mathcal{H}_{31}^{(2)}$ and $\mathcal{H}_{43}^{(2)}$ can be expressed in terms of harmonic polylogarithms and are discussed in Appendix A.

The higher orders in $\varepsilon$ of some of the master integrals are needed due to the appearance of so-called spurious poles which arise while solving the linear system of IBP equations. An alternative approach is to find a different set of master integrals through the method of $\varepsilon$-finite basis [38] which exploits the fact that the choice of the master integrals is not unique and which requires only the evaluation of the master integrals up to the order $\varepsilon^{0}$.

The master integrals constitute an essential input of our calculation, so we have checked the results using traditional Feynman parametrizations, partially analytically and partially through the numerical evaluation of the resulting Feynman integrals in order to ensure their correctness.

\section{Results}

\subsection{Matching factors}

In this section we give the conversion factors up to two-loop order in perturbative QCD for the fermion field and the mass parameter. This is accomplished as previously described in Section 2, For this purpose we decompose the matching factors into a part which depends on the gauge parameter and a part which is gauge parameter free,

$$
C_{y}^{x}=C_{y, L}^{x}+C_{y, \xi}^{x}, \quad x \in\left\{\mathrm{RI} / \mathrm{SMOM}, \mathrm{RI} / \mathrm{SMOM}_{\gamma_{\mu}}\right\}, \quad y \in\{q, m\},
$$

where $C_{y, \xi}^{x}$ contains all the terms which depend on the gauge parameter $\xi$, whereas $C_{y, L}^{x}$ is the conversion factor in the Landau gauge. In the following we present the contributions in the Landau gauge; for completeness, we also provide the gauge dependent terms in Appendix B. The one-loop results have been determined in Ref. [11].

Let us start with the conversion factors for the fermion field of Eqs. (41) and (5). They are important for the light quark mass renormalization procedure in Eq. (2), but also enter in the renormalization of other interesting quantities, i.e. any multiquark operator in such schemes, like, for example, the $B_{K}$ parameter. With the help of Ward-Takahashi identities it has been shown in Ref. [11] that $C_{q}^{\mathrm{RI} / \mathrm{SMOM}}=C_{q}^{\mathrm{RI}^{\prime} / \mathrm{MOM}}$. We have checked that our order $\alpha_{s}^{2}$ result for $C_{q}^{\mathrm{RI} / \mathrm{SMOM}}$ is in agreement with $C_{q}^{\mathrm{RI} / \mathrm{MOM}}$ from Ref. [8]. The fermion field matching factor in the RI/SMOM$\gamma_{\mu}$ scheme at two-loop order is new. The result is given by

$$
\begin{aligned}
C_{q, L}^{\mathrm{RI} / \mathrm{SMOM}_{\gamma \mu}} & =1+\left(\frac{\alpha_{s}}{4 \pi}\right) C_{F}+\left(\frac{\alpha_{s}}{4 \pi}\right)^{2}\left\{C_{F} n_{f} T_{F} \frac{5}{18}\right. \\
+C_{F}^{2} & {\left[-\frac{1}{8}+\frac{13 \pi^{2}}{3}+\frac{29 \pi^{3}}{162 \sqrt{3}}+\frac{2 \pi^{4}}{81}-2 \zeta_{3}+\frac{2 \pi \log (3)}{\sqrt{3}}-\frac{\pi \log ^{2}(3)}{6 \sqrt{3}}\right.}
\end{aligned}
$$




$$
\begin{gathered}
-24 s_{2}\left(\frac{\pi}{6}\right)+48 s_{2}\left(\frac{\pi}{2}\right)+40 s_{3}\left(\frac{\pi}{6}\right)-32 s_{3}\left(\frac{\pi}{2}\right)-\frac{13}{2} \Psi^{\prime}\left(\frac{1}{3}\right) \\
\left.\quad-\frac{8 \pi^{2}}{27} \Psi^{\prime}\left(\frac{1}{3}\right)+\frac{2}{9} \Psi^{\prime}\left(\frac{1}{3}\right)^{2}+\frac{1}{36} \Psi^{\prime \prime \prime}\left(\frac{1}{3}\right)\right] \\
+C_{A} C_{F}\left[-\frac{31}{72}-\frac{41 \pi^{2}}{18}-\frac{145 \pi^{3}}{1296 \sqrt{3}}-\frac{7 \pi^{4}}{81}-\zeta_{3}-\frac{5 \pi \log (3)}{4 \sqrt{3}}+\frac{5 \pi \log ^{2}(3)}{48 \sqrt{3}}\right. \\
+15 s_{2}\left(\frac{\pi}{6}\right)-30 s_{2}\left(\frac{\pi}{2}\right)-25 s_{3}\left(\frac{\pi}{6}\right)+20 s_{3}\left(\frac{\pi}{2}\right)+\frac{41}{12} \Psi^{\prime}\left(\frac{1}{3}\right) \\
\left.\left.+\frac{4 \pi^{2}}{27} \Psi^{\prime}\left(\frac{1}{3}\right)-\frac{1}{9} \Psi^{\prime}\left(\frac{1}{3}\right)^{2}+\frac{1}{72} \Psi^{\prime \prime \prime}\left(\frac{1}{3}\right)\right]\right\}+\mathcal{O}\left(\alpha_{s}^{3}\right) \\
\stackrel{N_{c}=3}{\simeq} 1+\left(\frac{\alpha_{s}}{4 \pi}\right) 1.333333333+\left(\frac{\alpha_{s}}{4 \pi}\right)^{2}\left[9.59901080+0.1851851852 n_{f}\right]+\mathcal{O}\left(\alpha_{s}^{3}\right) .
\end{gathered}
$$

The symbols $C_{F}$ and $C_{A}$ denote the Casimir operators of the fundamental and adjoint representations of $\mathrm{SU}\left(N_{c}\right)$. The normalization of the trace in the fundamental representation of $\mathrm{SU}\left(N_{c}\right)$ is given by $T_{F}=1 / 2$. For the number of colors $N_{c}=3$ one obtains $C_{F}=4 / 3$ and $C_{A}=3$. The number of active fermions is given by $n_{f}$.

Starting from two-loop order the strong coupling constant $\alpha_{s}$ also has to be renormalized, and it has to be ensured that the Slavnov-Taylor identities, which provide relations among the different renormalization constants, are preserved. These relations guarantee that the renormalized coupling constant remains the same for the different interaction vertices of the QCD Lagrangian. Therefore, we perform the renormalization of $\alpha_{s}$ in the $\overline{\mathrm{MS}}$ scheme with $\alpha_{s, R}=Z_{g}^{-2} \mu^{-2 \varepsilon} \alpha_{s, B}$. When not considering the Landau gauge, we also have to perform a renormalization of the gauge parameter $\xi_{R}=Z_{\xi}^{-1} \xi_{B}$ with $Z_{\xi}=Z_{3}$, where the renormalization constant $Z_{3}$ is the one of the gluon field $G^{a, \mu}$ with $G_{R}^{a, \mu}=1 / \sqrt{Z_{3}} G_{B}^{a, \mu}$. We renormalize these quantities in the $\overline{\mathrm{MS}}$ scheme (see also Appendix $\mathrm{B}$ ). In general, the ghost field $c^{a}$ also needs to be renormalized, $c_{R}^{a}=1 / \sqrt{Z_{3}^{c}} c_{B}^{a}$, which can also be done in the $\overline{\mathrm{MS}}$ scheme, but is not needed within this calculation.

In complete analogy to Eq. (20), the mass conversion factor, required for the matching of the light quark masses determined in the RI/SMOM scheme to the $\overline{\mathrm{MS}}$ scheme, reads up to two-loop order in the Landau gauge $(\xi=0)$

$$
\begin{aligned}
C_{m, L}^{\mathrm{RI} / \mathrm{SMOM}}= & +\left(\frac{\alpha_{s}}{4 \pi}\right) C_{F}\left(\Psi^{\prime}\left(\frac{1}{3}\right)-\frac{2}{3} \pi^{2}-4\right) \\
+ & \left(\frac{\alpha_{s}}{4 \pi}\right)^{2}\left\{C_{F} T_{F} n_{f}\left[\frac{83}{6}+\frac{40 \pi^{2}}{27}-\frac{20}{9} \Psi^{\prime}\left(\frac{1}{3}\right)\right]\right. \\
+ & C_{F}^{2}\left[\frac{19}{8}+\frac{52 \pi^{2}}{9}+\frac{29 \pi^{3}}{162 \sqrt{3}}+\frac{76 \pi^{4}}{81}+3 \Sigma+4 \zeta_{3}+\frac{2 \pi \log (3)}{\sqrt{3}}\right. \\
& -\frac{26}{3} \Psi^{\prime}\left(\frac{1}{3}\right)-\frac{52 \pi^{2}}{27} \Psi^{\prime}\left(\frac{1}{3}\right)+\frac{13}{9} \Psi^{\prime}\left(\frac{1}{3}\right)^{2}-\frac{1}{9} \Psi^{\prime \prime \prime}\left(\frac{1}{3}\right) \\
& \left.-\frac{\pi \log ^{2}(3)}{6 \sqrt{3}}-24 s_{2}\left(\frac{\pi}{6}\right)+48 s_{2}\left(\frac{\pi}{2}\right)+40 s_{3}\left(\frac{\pi}{6}\right)-32 s_{3}\left(\frac{\pi}{2}\right)\right]
\end{aligned}
$$




$$
\begin{aligned}
+C_{A} C_{F}\left[\frac{7 \pi^{4}}{81}\right. & -\frac{1285}{24}-\frac{457 \pi^{2}}{54}-\frac{29 \pi^{3}}{324 \sqrt{3}}+\frac{5}{2} \Sigma+10 \zeta_{3}-\frac{\pi \log (3)}{\sqrt{3}} \\
& -\frac{5}{72} \Psi^{\prime \prime \prime}\left(\frac{1}{3}\right)+12 s_{2}\left(\frac{\pi}{6}\right)-24 s_{2}\left(\frac{\pi}{2}\right)-20 s_{3}\left(\frac{\pi}{6}\right)+16 s_{3}\left(\frac{\pi}{2}\right) \\
& \left.\left.+\frac{\pi \log ^{2}(3)}{12 \sqrt{3}}+\frac{457}{36} \Psi^{\prime}\left(\frac{1}{3}\right)+\frac{8 \pi^{2}}{27} \Psi^{\prime}\left(\frac{1}{3}\right)-\frac{2}{9} \Psi^{\prime}\left(\frac{1}{3}\right)^{2}\right]\right\}+\mathcal{O}\left(\alpha_{s}^{3}\right) \\
\stackrel{N_{c}=3}{\simeq} 1- & \left(\frac{\alpha_{s}}{4 \pi}\right) 0.6455188560-\left(\frac{\alpha_{s}}{4 \pi}\right)^{2}\left[22.60768757-4.013539470 n_{f}\right]+\mathcal{O}\left(\alpha_{s}^{3}\right),
\end{aligned}
$$

where the symbol $\Sigma$ is given in Appendix A. Similarly, we obtain, for the mass conversion factor up to two-loop order in the RI/SMOM $\gamma_{\mu}$ scheme,

$$
\begin{aligned}
C_{m, L}^{\mathrm{RI} / \mathrm{SMOM}_{\gamma_{\mu}}=} & 1+\left(\frac{\alpha_{s}}{4 \pi}\right) C_{F}\left(\Psi^{\prime}\left(\frac{1}{3}\right)-\frac{2}{3} \pi^{2}-5\right) \\
& +\left(\frac{\alpha_{s}}{4 \pi}\right)^{2}\left\{n_{f} C_{F} T_{F}\left[\frac{307}{18}+\frac{40 \pi^{2}}{27}-\frac{20}{9} \Psi^{\prime}\left(\frac{1}{3}\right)\right]\right. \\
+ & C_{F}^{2}\left[\frac{65}{8}+\frac{19 \pi^{2}}{9}+\frac{74 \pi^{4}}{81}+3 \Sigma+6 \zeta_{3}-\frac{19}{6} \Psi^{\prime}\left(\frac{1}{3}\right)-\frac{44 \pi^{2}}{27} \Psi^{\prime}\left(\frac{1}{3}\right)+\frac{11}{9} \Psi^{\prime}\left(\frac{1}{3}\right)^{2}\right. \\
& \left.-\frac{5}{36} \Psi^{\prime \prime \prime}\left(\frac{1}{3}\right)\right] \\
+ & C_{F} C_{A}\left[\frac{14 \pi^{4}}{81}-\frac{2281}{36}-\frac{167 \pi^{2}}{27}+\frac{29 \pi^{3}}{1296 \sqrt{3}}+\frac{5}{2} \Sigma+14 \zeta_{3}+\frac{\pi \log (3)}{4 \sqrt{3}}\right. \\
& -\frac{\pi \log ^{2}(3)}{48 \sqrt{3}}+\frac{167}{18} \Psi^{\prime}\left(\frac{1}{3}\right)+\frac{4 \pi^{2}}{27} \Psi^{\prime}\left(\frac{1}{3}\right)-\frac{1}{9} \Psi^{\prime}\left(\frac{1}{3}\right)^{2}-3 s_{2}\left(\frac{\pi}{6}\right)+6 s_{2}\left(\frac{\pi}{2}\right) \\
& \left.\left.-\frac{1}{12} \Psi^{\prime \prime \prime}\left(\frac{1}{3}\right)+5 s_{3}\left(\frac{\pi}{6}\right)-4 s_{3}\left(\frac{\pi}{2}\right)\right]\right\}+\mathcal{O}\left(\alpha_{s}^{3}\right) \\
N_{c=3} 1- & \left(\frac{\alpha_{s}}{4 \pi}\right) 1.978852189-\left(\frac{\alpha_{s}}{4 \pi}\right)^{2}\left[55.03243483-6.161687618 n_{f}\right]+\mathcal{O}\left(\alpha_{s}^{3}\right) .
\end{aligned}
$$

We want to mention that the matching factors in Eqs. (21) and (22) are related to conversion factors of quark bilinear operators. As shown in Ref. [1] Ward-Takahashi identities allow us to write the matching factor $C_{P}^{x}$, which converts the pseudoscalar operator renormalized in the $x$ scheme to the $\overline{\mathrm{MS}}$ scheme, as the inverse of $C_{m}^{x}$ for $x \in\{\mathrm{RI} / \mathrm{SmOM}$, RI/SMOM $\left.\gamma_{\gamma_{\mu}}\right\}$. Furthermore, the latter are also related to the corresponding matching factor of the scalar operator $C_{S}^{x}=C_{P}^{x}=1 / C_{m}^{x}, x \in\left\{\mathrm{RI} / \mathrm{Smom}, \mathrm{RI} / \mathrm{Smom}_{\gamma_{\mu}}\right\}$. For the definition of these matching factors we adopt the same notations as in Ref. [11]. For completeness we give here also the result for the matching factor $C_{T}^{x}$ of the tensor operator and again disentangle $C_{T}^{x}$ into its Landau gauge $(\xi=0)$ and its gauge dependent parts in complete analogy to the decomposition of Eq. (19) for $y=T$. The Landau gauge component is given by

$$
C_{T, L}^{\mathrm{RI} / \mathrm{SMOM}}=1+\left(\frac{\alpha_{s}}{4 \pi}\right) C_{F}\left(\frac{1}{3} \Psi^{\prime}\left(\frac{1}{3}\right)-\frac{2}{9} \pi^{2}-\frac{4}{3}\right)
$$




$$
\begin{aligned}
& +\left(\frac{\alpha_{s}}{4 \pi}\right)^{2}\left\{C_{F} T_{F} n_{f}\left[\frac{473}{54}-\frac{20}{27} \Psi^{\prime}\left(\frac{1}{3}\right)+\frac{40}{81} \pi^{2}\right]\right. \\
+C_{F}^{2}[ & \frac{599}{24}+\frac{178}{9} \Psi^{\prime}\left(\frac{1}{3}\right)-\frac{4}{27} \Psi^{\prime}\left(\frac{1}{3}\right)^{2}-\frac{356}{27} \pi^{2}+\frac{16}{81} \Psi^{\prime}\left(\frac{1}{3}\right) \pi^{2}+\frac{32}{243} \pi^{4} \\
& -\frac{2}{27} \Psi^{\prime \prime \prime}\left(\frac{1}{3}\right)-240 s_{2}\left(\frac{\pi}{2}\right)+120 s_{2}\left(\frac{\pi}{6}\right)+160 s_{3}\left(\frac{\pi}{2}\right)-200 s_{3}\left(\frac{\pi}{6}\right) \\
& \left.+\Sigma-\frac{10 \log (3) \pi}{\sqrt{3}}+\frac{5 \log ^{2}(3) \pi}{6 \sqrt{3}}-\frac{145 \pi^{3}}{162 \sqrt{3}}-12 \zeta_{3}\right] \\
+C_{A} C_{F} & {\left[-\frac{8491}{216}-\frac{755}{108} \Psi^{\prime}\left(\frac{1}{3}\right)+\frac{2}{27} \Psi^{\prime}\left(\frac{1}{3}\right)^{2}+\frac{755}{162} \pi^{2}-\frac{8}{81} \Psi^{\prime}\left(\frac{1}{3}\right) \pi^{2}\right.} \\
& +\frac{35}{243} \pi^{4}-\frac{1}{24} \Psi^{\prime \prime \prime}\left(\frac{1}{3}\right)+128 s_{2}\left(\frac{\pi}{2}\right)-64 s_{2}\left(\frac{\pi}{6}\right)-\frac{256}{3} s_{3}\left(\frac{\pi}{2}\right)+\frac{320}{3} s_{3}\left(\frac{\pi}{6}\right) \\
& \left.\left.+\frac{5}{6} \Sigma+\frac{16 \log (3) \pi}{3 \sqrt{3}}-\frac{4 \log ^{2}(3) \pi}{9 \sqrt{3}}+\frac{116 \pi^{3}}{243 \sqrt{3}}+\frac{50}{3} \zeta_{3}\right]\right\}+\mathcal{O}\left(\alpha_{s}^{3}\right) \\
N_{c}=3 & -\left(\frac{\alpha_{s}}{4 \pi}\right) 0.21517295-\left(\frac{\alpha_{s}}{4 \pi}\right)^{2}\left[43.38395007-4.10327859 n_{f}\right]+\mathcal{O}\left(\alpha_{s}^{3}\right)
\end{aligned}
$$

and

$$
\begin{aligned}
C_{T, L}^{\mathrm{RI} / \mathrm{SMOM}_{\gamma \mu}}= & 1+\left(\frac{\alpha_{s}}{4 \pi}\right) C_{F}\left(\frac{1}{3} \Psi^{\prime}\left(\frac{1}{3}\right)-\frac{2}{9} \pi^{2}-\frac{1}{3}\right) \\
& +\left(\frac{\alpha_{s}}{4 \pi}\right)^{2}\left\{C_{F} T_{F} n_{f}\left[\frac{299}{54}-\frac{20}{27} \Psi^{\prime}\left(\frac{1}{3}\right)+\frac{40}{81} \pi^{2}\right]\right. \\
+C_{F}^{2}[ & {\left[\frac{183}{8}+\frac{245}{18} \Psi^{\prime}\left(\frac{1}{3}\right)+\frac{2}{27} \Psi^{\prime}\left(\frac{1}{3}\right)^{2}-\frac{245}{27} \pi^{2}-\frac{8}{81} \Psi^{\prime}\left(\frac{1}{3}\right) \pi^{2}+\frac{38}{243} \pi^{4}\right.} \\
& -\frac{5}{108} \Psi^{\prime \prime \prime}\left(\frac{1}{3}\right)-192 s_{2}\left(\frac{\pi}{2}\right)+96 s_{2}\left(\frac{\pi}{6}\right)+128 s_{3}\left(\frac{\pi}{2}\right)-160 s_{3}\left(\frac{\pi}{6}\right)+\Sigma \\
& \left.-\frac{8 \log (3) \pi}{\sqrt{3}}+\frac{2 \log ^{2}(3) \pi}{3 \sqrt{3}}-\frac{58 \pi^{3}}{81 \sqrt{3}}-14 \zeta_{3}\right] \\
+C_{A} & C_{F}\left[-\frac{3185}{108}-\frac{193}{54} \Psi^{\prime}\left(\frac{1}{3}\right)-\frac{1}{27} \Psi^{\prime}\left(\frac{1}{3}\right)^{2}+\frac{193}{81} \pi^{2}+\frac{4}{81} \Psi^{\prime}\left(\frac{1}{3}\right) \pi^{2}+\frac{14}{243} \pi^{4}\right. \\
& -\frac{1}{36} \Psi^{\prime \prime \prime}\left(\frac{1}{3}\right)+98 s_{2}\left(\frac{\pi}{2}\right)-49 s_{2}\left(\frac{\pi}{6}\right)-\frac{196}{3} s_{3}\left(\frac{\pi}{2}\right)+\frac{245}{3} s_{3}\left(\frac{\pi}{6}\right)+\frac{5}{6} \Sigma \\
& \left.\left.+\frac{49 \log (3) \pi}{12 \sqrt{3}}-\frac{49 \log ^{2}(3) \pi}{144 \sqrt{3}}+\frac{1421 \pi^{3}}{3888 \sqrt{3}}+\frac{38}{3} \zeta_{3}\right]\right\}+\mathcal{O}\left(\alpha_{s}^{3}\right) \\
N_{c}=3 & +\left(\frac{\alpha_{s}}{4 \pi}\right) 1.11816038-\left(\frac{\alpha_{s}}{4 \pi}\right)^{2}\left[8.607630493-1.955130440 n_{f}\right]+\mathcal{O}\left(\alpha_{s}^{3}\right) .
\end{aligned}
$$

The gauge dependent component can be found in Appendix $\mathrm{B}$. The one-loop order in the $\mathrm{RI} / \mathrm{SMOM}$ scheme has already been computed in Ref. [11]. The result in the RI'/MOM scheme is available up to three-loop order in Ref. [9]. 


\subsection{Comparison with results using exceptional momenta}

In order to analyze the quality of the new two-loop order of the mass conversion factors in Eqs. (21) and (22), we compare them to the results of the traditional $\mathrm{RI}^{\prime} / \mathrm{MOM}$ and RI/MOM schemes. For this purpose we evaluate the matching factors numerically, again in the Landau gauge, for $n_{f}=3$ flavors. For the strong coupling constant we insert $\alpha_{s} / \pi=0.11$. The results in the RI/SMOM versus the $\mathrm{RI}^{\prime} / \mathrm{MOM}$ scheme for $N_{c}=3 \mathrm{read}$

$$
\begin{aligned}
C_{m, L}^{\mathrm{RI} / \mathrm{SMOM}} & =1-0.0161380 \ldots-0.00660442 \ldots \\
C_{m, L}^{\mathrm{RI} / \mathrm{MOM}} & =1-0.1333333 \ldots-0.07585848 \ldots-0.0556959 \ldots
\end{aligned}
$$

where each term stands for the next order in perturbative QCD, i.e. the leading, nextto-leading, and next-to-next-to-leading order results. Similarly follow the results for the $\mathrm{RI} / \mathrm{SMOM}_{\gamma_{\mu}}$ versus the RI/MOM scheme

$$
\begin{aligned}
C_{m, L}^{\mathrm{RI} / \mathrm{SMOM}_{\gamma \mu}} & =1-0.0494713 \ldots-0.0228421 \ldots, \\
C_{m, L}^{\mathrm{RI} / \mathrm{MOM}} & =1-0.1333333 \ldots-0.0815876 \ldots-0.0602759 \ldots
\end{aligned}
$$

Both the RI/MOM and $\mathrm{RI}^{\prime} / \mathrm{MOM}$ schemes are known to three-loop order, where we have taken the results of Ref. [8]. One can observe that the matching factors of the schemes with a symmetric subtraction point in Eqs. (25) and (27) show a much better convergence behavior than the ones in the $\mathrm{RI}^{\prime} / \mathrm{MOM}$ and RI/MOM schemes of Eqs. (26) and (28) which are characterized by an exceptional momentum-subtraction point. This observation has already been made in Ref. [11] at one-loop order and is now confirmed at the two-loop level. The size of the three-loop corrections is about $6 \%$ of the leading order result for both the RI/MOM and $\mathrm{RI}^{\prime} / \mathrm{MOM}$ schemes, whereas in the RI/SMOM scheme the two-loop result is already significantly smaller, being of about only 7 per mill 2 of the leading order one. Similarly, for the RI/SMOM $\gamma_{\mu}$ scheme the size of the two-loop term is of about $2 \%$, which is also smaller than the three-loop terms of the two schemes with an exceptional subtraction point. The use of these new results in light up-, down-, and strange-quark mass determinations in the context of NPR will allow us to reduce the uncertainties related to the matching procedure to the $\overline{\mathrm{MS}}$ scheme, due to a smaller truncation error of the perturbative series, which will significantly reduce the error of these light quark masses obtained in this approach.

\footnotetext{
${ }^{1}$ The choice of this value corresponds approximately to the value of $\alpha_{s} / \pi$ at a scale of $2 \mathrm{GeV}$ and is sufficient to illustrate the effect of the new results. It allows the reader to easily replace it by her/his own value of $\alpha_{s}$.

${ }^{2}$ Since the percentage correction of the two-loop term in the RI/SMOM scheme is very small, its size is more sensitive to the exact value of $\alpha_{s}$ than in the other schemes. Thus a slightly smaller value of $\alpha_{s} / \pi$ leads to a slightly smaller correction of about $0.6 \%$ for the RI/SMOM scheme.
} 


\subsection{Anomalous dimensions}

The mass anomalous dimension of the RI/SMOM and RI/SMOM $\gamma_{\gamma_{\mu}}$ schemes is used to run the quark masses to different energy scales. It is defined by

$$
\gamma_{m}^{x}=\frac{d \log m^{x}(\mu)}{d \log \mu^{2}}=-\gamma_{m}^{(0), x} \frac{\alpha_{s}}{4 \pi}-\gamma_{m}^{(1), x}\left(\frac{\alpha_{s}}{4 \pi}\right)^{2}-\gamma_{m}^{(2), x}\left(\frac{\alpha_{s}}{4 \pi}\right)^{3}+\mathcal{O}\left(\alpha_{s}^{4}\right),
$$

where the superscript $x$ stands, similar to Eq. (19), for the scheme with $x \in$ \{RI/SMOM, RI/SMOM $\left.\gamma_{\mu}, \overline{\mathrm{MS}}\right\}$. Both mass anomalous dimensions are related to the $\overline{\mathrm{MS}}$ mass anomalous dimension $\gamma_{m}^{\overline{\mathrm{MS}}}$ through the conversion factors $C_{m}^{x}$ presented in Section 4.1 (see Ref. 8]). In the Landau gauge holds

$$
\gamma_{m}^{x}=\gamma_{m}^{\overline{\mathrm{MS}}}-\beta \frac{\partial \log C_{m, L}^{x}}{\partial \frac{\alpha_{s}}{4 \pi}}, \quad x \in\left\{\mathrm{RI} / \mathrm{SMOM}, \mathrm{RI} / \mathrm{SMOM}_{\gamma_{\mu}}\right\},
$$

where the QCD $\beta$ function and $\gamma_{m}^{\overline{\mathrm{MS}}}$ are given, for completeness, in Appendix C. The one-loop mass anomalous dimensions in the RI/SMOM and RI/SMOM $\gamma_{\mu}$ schemes are equal to the one in the $\overline{\mathrm{MS}}$ scheme; the mass anomalous dimension at two-loop order has been determined in Ref. [11] and is also given in Appendix D. The three-loop result is presented here and reads, in the Landau gauge in the RI/SMOM scheme 3

$$
\begin{aligned}
-\gamma_{m}^{(2), \mathrm{RI} / \mathrm{SMOM}}= & -\frac{129}{2} C_{F}^{3}+C_{A}^{2} C_{F}\left[-\frac{29357}{54}-\frac{5639 \pi^{2}}{81}-\frac{319 \pi^{3}}{486 \sqrt{3}}+\frac{154 \pi^{4}}{243}+\frac{55}{3} \Sigma\right. \\
- & \frac{55}{108} \Psi^{\prime \prime \prime}\left(\frac{1}{3}\right)-\frac{22 \pi \log (3)}{3 \sqrt{3}}+\frac{11 \pi \log ^{2}(3)}{18 \sqrt{3}}+\frac{5639}{54} \Psi^{\prime}\left(\frac{1}{3}\right)+\frac{176 \pi^{2}}{81} \Psi^{\prime}\left(\frac{1}{3}\right) \\
& \left.-\frac{44}{27} \Psi^{\prime}\left(\frac{1}{3}\right)^{2}+88 s_{2}\left(\frac{\pi}{6}\right)-176 s_{2}\left(\frac{\pi}{2}\right)-\frac{440}{3} s_{3}\left(\frac{\pi}{6}\right)+\frac{352}{3} s_{3}\left(\frac{\pi}{2}\right)+\frac{220}{3} \zeta_{3}\right] \\
+n_{f} T_{F} & \left\{C _ { F } ^ { 2 } \left[77-\frac{152 \pi^{2}}{27}-\frac{116 \pi^{3}}{243 \sqrt{3}}-\frac{464 \pi^{4}}{243}-8 \Sigma+\frac{8}{27} \Psi^{\prime \prime \prime}\left(\frac{1}{3}\right)+\frac{4 \pi \log ^{2}(3)}{9 \sqrt{3}}\right.\right. \\
+ & \frac{76}{9} \Psi^{\prime}\left(\frac{1}{3}\right)+\frac{272 \pi^{2}}{81} \Psi^{\prime}\left(\frac{1}{3}\right)-\frac{68}{27} \Psi^{\prime}\left(\frac{1}{3}\right)^{2}-\frac{16 \pi \log (3)}{3 \sqrt{3}}+64 s_{2}\left(\frac{\pi}{6}\right)-128 s_{2}\left(\frac{\pi}{2}\right) \\
& \left.-\frac{320}{3} s_{3}\left(\frac{\pi}{6}\right)+\frac{256}{3} s_{3}\left(\frac{\pi}{2}\right)-\frac{176}{3} \zeta_{3}\right]+C_{A} C_{F}\left[\frac{7870}{27}+\frac{3068 \pi^{2}}{81}+\frac{58 \pi^{3}}{243 \sqrt{3}}-\frac{56 \pi^{4}}{243}\right. \\
& -\frac{20}{3} \Sigma+\frac{8 \pi \log (3)}{3 \sqrt{3}}-\frac{2 \pi \log ^{2}(3)}{9 \sqrt{3}}-\frac{1534}{27} \Psi^{\prime}\left(\frac{1}{3}\right)-\frac{64 \pi^{2}}{81} \Psi^{\prime}\left(\frac{1}{3}\right)+\frac{16}{27} \Psi^{\prime}\left(\frac{1}{3}\right)^{2} \\
+ & \left.\left.\frac{5}{27} \Psi^{\prime \prime \prime}\left(\frac{1}{3}\right)-32 s_{2}\left(\frac{\pi}{6}\right)+64 s_{2}\left(\frac{\pi}{2}\right)+\frac{160}{3} s_{3}\left(\frac{\pi}{6}\right)-\frac{128}{3} s_{3}\left(\frac{\pi}{2}\right)+\frac{64}{3} \zeta_{3}\right]\right\} \\
+C_{A} C_{F}^{2} & {\left[-9+\frac{616 \pi^{2}}{27}+\frac{319 \pi^{3}}{243 \sqrt{3}}+\frac{1276 \pi^{4}}{243}+22 \Sigma+\frac{44 \pi \log (3)}{3 \sqrt{3}}-\frac{11 \pi \log ^{2}(3)}{9 \sqrt{3}}-\frac{308}{9} \Psi^{\prime}\left(\frac{1}{3}\right)\right.}
\end{aligned}
$$

\footnotetext{
${ }^{3}$ The minus sign on the left-hand side of Eqs. (31), (32) and (35) is correct, but was missing in the first arXiv version. It is needed to be consistent with the definition in Eqs.(29) and (33), respectively; see also the note added.
} 


$$
\begin{aligned}
& -\frac{748 \pi^{2}}{81} \Psi^{\prime}\left(\frac{1}{3}\right)+\frac{187}{27} \Psi^{\prime}\left(\frac{1}{3}\right)^{2}-\frac{22}{27} \Psi^{\prime \prime \prime}\left(\frac{1}{3}\right)-176 s_{2}\left(\frac{\pi}{6}\right)+352 s_{2}\left(\frac{\pi}{2}\right)+\frac{880}{3} s_{3}\left(\frac{\pi}{6}\right) \\
& \left.-\frac{704}{3} s_{3}\left(\frac{\pi}{2}\right)+\frac{88}{3} \zeta_{3}\right]+C_{F} n_{f}^{2} T_{F}^{2}\left[-\frac{856}{27}-\frac{320 \pi^{2}}{81}+\frac{160}{27} \Psi^{\prime}\left(\frac{1}{3}\right)\right] .
\end{aligned}
$$

In the RI/SMOM $\gamma_{\mu}$ scheme the two-loop order is again available in Ref. [1] (see also Appendix (D) and the three-loop result in the Landau gauge is given by ${ }^{3}$

$$
\begin{aligned}
& -\gamma_{m}^{(2), \mathrm{RI} / \mathrm{SMOM}_{\gamma_{\mu}}}=-\frac{129}{2} C_{F}^{3}+C_{F}^{2} C_{A}\left[\frac{1}{6}-\frac{242 \pi^{2}}{27}+\frac{121}{9} \Psi^{\prime}\left(\frac{1}{3}\right)-\frac{572 \pi^{2}}{81} \Psi^{\prime}\left(\frac{1}{3}\right)\right. \\
& \left.+\frac{143}{27} \Psi^{\prime}\left(\frac{1}{3}\right)^{2}+44 \zeta_{3}+\frac{1232 \pi^{4}}{243}+22 \Sigma-\frac{55}{54} \Psi^{\prime \prime \prime}\left(\frac{1}{3}\right)\right] \\
& +C_{F} C_{A}^{2}\left[-\frac{67715}{108}-\frac{4286 \pi^{2}}{81}+\frac{319 \pi^{3}}{1944 \sqrt{3}}+\frac{308 \pi^{4}}{243}+\frac{55}{3} \Sigma-\frac{11}{18} \Psi^{\prime \prime \prime}\left(\frac{1}{3}\right)\right. \\
& +\frac{11 \pi \log (3)}{6 \sqrt{3}}-\frac{11 \pi \log ^{2}(3)}{72 \sqrt{3}}+\frac{2143}{27} \Psi^{\prime}\left(\frac{1}{3}\right)+\frac{88 \pi^{2}}{81} \Psi^{\prime}\left(\frac{1}{3}\right)-\frac{22}{27} \Psi^{\prime}\left(\frac{1}{3}\right)^{2} \\
& \left.-22 s_{2}\left(\frac{\pi}{6}\right)+44 s_{2}\left(\frac{\pi}{2}\right)+\frac{110}{3} s_{3}\left(\frac{\pi}{6}\right)-\frac{88}{3} s_{3}\left(\frac{\pi}{2}\right)+\frac{308}{3} \zeta_{3}\right] \\
& +n_{f} T_{F}\left\{C _ { F } ^ { 2 } \left[\frac{233}{3}+\frac{160 \pi^{2}}{27}-\frac{448 \pi^{4}}{243}-8 \Sigma+\frac{10}{27} \Psi^{\prime \prime \prime}\left(\frac{1}{3}\right)-\frac{80}{9} \Psi^{\prime}\left(\frac{1}{3}\right)+\frac{208 \pi^{2}}{81} \Psi^{\prime}\left(\frac{1}{3}\right)\right.\right. \\
& \left.-\frac{52}{27} \Psi^{\prime}\left(\frac{1}{3}\right)^{2}-64 \zeta_{3}\right] \\
& +C_{A} C_{F}\left[\frac{9395}{27}+\frac{2576 \pi^{2}}{81}-\frac{29 \pi^{3}}{486 \sqrt{3}}-\frac{112 \pi^{4}}{243}-\frac{20}{3} \Sigma+\frac{2}{9} \Psi^{\prime \prime \prime}\left(\frac{1}{3}\right)-\frac{2 \pi \log (3)}{3 \sqrt{3}}\right. \\
& +\frac{\pi \log ^{2}(3)}{18 \sqrt{3}}-\frac{1288}{27} \Psi^{\prime}\left(\frac{1}{3}\right)-\frac{32 \pi^{2}}{81} \Psi^{\prime}\left(\frac{1}{3}\right)+\frac{8}{27} \Psi^{\prime}\left(\frac{1}{3}\right)^{2}+8 s_{2}\left(\frac{\pi}{6}\right) \\
& \left.\left.-16 s_{2}\left(\frac{\pi}{2}\right)-\frac{40}{3} s_{3}\left(\frac{\pi}{6}\right)+\frac{32}{3} s_{3}\left(\frac{\pi}{2}\right)+\frac{32}{3} \zeta_{3}\right]\right\} \\
& +n_{f}^{2} T_{F}^{2} C_{F}\left[\frac{160}{27} \Psi^{\prime}\left(\frac{1}{3}\right)-\frac{1088}{27}-\frac{320 \pi^{2}}{81}\right] .
\end{aligned}
$$

Similarly to the mass anomalous dimension we define the anomalous dimension of the fermion field as

$$
\gamma_{q}^{x}=2 \frac{d \log \Psi_{R, x}}{d \log \mu^{2}}=-\gamma_{q}^{(0), x} \frac{\alpha_{s}}{4 \pi}-\gamma_{q}^{(1), x}\left(\frac{\alpha_{s}}{4 \pi}\right)^{2}-\gamma_{q}^{(2), x}\left(\frac{\alpha_{s}}{4 \pi}\right)^{3}+\mathcal{O}\left(\alpha_{s}^{4}\right),
$$

where the renormalized and bare fields are connected by $\Psi_{R}=\sqrt{Z_{q}} \Psi_{0}$. The relation between the $\overline{\mathrm{MS}}$ fermion field anomalous dimension and the ones found in the RI/SMOM and $\mathrm{RI} / \mathrm{SMOM}_{\gamma_{\mu}}$ schemes is, in the Landau gauge, again given by [8]

$$
\gamma_{q}^{x}=\gamma_{q}^{\overline{\mathrm{MS}}}-\beta \frac{\partial C_{q, L}^{x}}{\partial \frac{\alpha_{s}}{4 \pi}}, \quad x \in\left\{\mathrm{RI} / \mathrm{SMOM}, \mathrm{RI} / \mathrm{SMOM}_{\gamma_{\mu}}, \overline{\mathrm{MS}}\right\}
$$


In the RI/SMOM scheme $\gamma_{q}^{\mathrm{RI} / \mathrm{SMOM}}$ is equal to $\gamma_{q}^{\mathrm{RI} / \mathrm{MOM}}$ to all loop orders in perturbation theory, which has been shown in Ref. [11]. We have reaffirmed this equivalence to $\mathcal{O}\left(\alpha_{s}^{3}\right)$ by comparing our results with those of Ref. [8]. For the sake of brevity, we omit this result since $\gamma_{q}^{\mathrm{RI}^{\prime} / \mathrm{MOM}}$, at the three-loop level, can be found in Ref. 8. However, such an equality is no longer true in the RI/SMOM ${ }_{\gamma_{\mu}}$ scheme. Therefore, we provide the threeloop contribution to the fermion field anomalous dimension in this scheme in the Landau gauge below ${ }^{3}$

$$
\begin{aligned}
&-\gamma_{q}^{(2), \mathrm{RI} / \mathrm{SMOM}_{\gamma_{\mu}}=}-\frac{3}{2} C_{F}^{3}+C_{F}^{2} C_{A}\left[\frac{187}{6}+\frac{286 \pi^{2}}{9}+\frac{319 \pi^{3}}{243 \sqrt{3}}+\frac{44 \pi^{4}}{243}+\frac{44 \pi \log (3)}{3 \sqrt{3}}\right. \\
&- \frac{11 \pi \log ^{2}(3)}{9 \sqrt{3}}-\frac{143}{3} \Psi^{\prime}\left(\frac{1}{3}\right)-\frac{176 \pi^{2}}{81} \Psi^{\prime}\left(\frac{1}{3}\right)+\frac{44}{27} \Psi^{\prime}\left(\frac{1}{3}\right)^{2}+\frac{11}{54} \Psi^{\prime \prime \prime}\left(\frac{1}{3}\right) \\
&\left.-176 s_{2}\left(\frac{\pi}{6}\right)+352 s_{2}\left(\frac{\pi}{2}\right)+\frac{880}{3} s_{3}\left(\frac{\pi}{6}\right)-\frac{704}{3} s_{3}\left(\frac{\pi}{2}\right)-\frac{80}{3} \zeta_{3}\right] \\
&+ C_{F} C_{A}^{2}\left[-\frac{23933}{432}-\frac{451 \pi^{2}}{27}-\frac{1595 \pi^{3}}{1944 \sqrt{3}}-\frac{154 \pi^{4}}{243}-\frac{55 \pi \log (3)}{6 \sqrt{3}}+\frac{55 \pi \log ^{2}(3)}{72 \sqrt{3}}\right. \\
&+\frac{451}{18} \Psi^{\prime}\left(\frac{1}{3}\right)+\frac{88 \pi^{2}}{81} \Psi^{\prime}\left(\frac{1}{3}\right)-\frac{22}{27} \Psi^{\prime}\left(\frac{1}{3}\right)^{2}+\frac{11}{108} \Psi^{\prime \prime \prime}\left(\frac{1}{3}\right)+110 s_{2}\left(\frac{\pi}{6}\right)-220 s_{2}\left(\frac{\pi}{2}\right) \\
&\left.-\frac{550}{3} s_{3}\left(\frac{\pi}{6}\right)+\frac{440}{3} s_{3}\left(\frac{\pi}{2}\right)+\frac{31}{24} \zeta_{3}\right] \\
&+ n_{f} T_{F}\left\{C _ { F } ^ { 2 } \left[-\frac{16}{3}-\frac{104 \pi^{2}}{9}-\frac{116 \pi^{3}}{243 \sqrt{3}}-\frac{16 \pi^{4}}{243}-\frac{16 \pi \log (3)}{3 \sqrt{3}}+\frac{4 \pi \log ^{2}(3)}{9 \sqrt{3}}+\frac{52}{3} \Psi^{\prime}\left(\frac{1}{3}\right)\right.\right. \\
&+\frac{64 \pi^{2}}{81} \Psi^{\prime}\left(\frac{1}{3}\right)-\frac{16}{27} \Psi^{\prime}\left(\frac{1}{3}\right)^{2}-\frac{2}{27} \Psi^{\prime \prime \prime}\left(\frac{1}{3}\right)+64 s_{2}\left(\frac{\pi}{6}\right)-128 s_{2}\left(\frac{\pi}{2}\right)-\frac{320}{3} s_{3}\left(\frac{\pi}{6}\right) \\
&\left.+\frac{256}{3} s_{3}\left(\frac{\pi}{2}\right)+\frac{16}{3} \zeta_{3}\right] \\
&+ C_{F} C_{A}\left[\frac{767}{27}+\frac{164 \pi^{2}}{27}+\frac{145 \pi^{3}}{486 \sqrt{3}}+\frac{56 \pi^{4}}{243}+\frac{10 \pi \log (3)}{3 \sqrt{3}}-\frac{5 \pi \log ^{2}(3)}{18 \sqrt{3}}-\frac{82}{9} \Psi^{\prime}\left(\frac{1}{3}\right)\right. \\
&-\frac{32 \pi^{2}}{81} \Psi^{\prime}\left(\frac{1}{3}\right)+\frac{8}{27} \Psi^{\prime}\left(\frac{1}{3}\right)^{2}-\frac{1}{27} \Psi^{\prime \prime \prime}\left(\frac{1}{3}\right)-40 s_{2}\left(\frac{\pi}{6}\right)+80 s_{2}\left(\frac{\pi}{2}\right)+\frac{200}{3} s_{3}\left(\frac{\pi}{6}\right) \\
&\left.\left.-\frac{160}{3} s_{3}\left(\frac{\pi}{2}\right)+\frac{8}{3} \zeta_{3}\right]\right\}-n_{f}^{2} C_{F} T_{F}^{2} \frac{80}{27} .
\end{aligned}
$$

The one- and two-loop contributions are again given in Ref. [11] and can also be found in Appendix D,

The anomalous dimension of the tensor operator is defined by

$$
\gamma_{T}^{x}=\frac{d \log Z_{T}^{x}}{d \log \mu^{2}}=-\gamma_{T}^{(0), x} \frac{\alpha_{s}}{4 \pi}-\gamma_{T}^{(1), x}\left(\frac{\alpha_{s}}{4 \pi}\right)^{2}-\gamma_{T}^{(2), x}\left(\frac{\alpha_{s}}{4 \pi}\right)^{3}+\mathcal{O}\left(\alpha_{s}^{4}\right),
$$

where the renormalized and bare operators are related by the renormalization constant $Z_{\hat{O}}^{x}$ with $\hat{O}_{R}^{x}=Z_{\hat{O}}^{x} \hat{O}_{B}$ for $\hat{O}=T$ and $x \in\left\{\mathrm{RI} / \mathrm{SmOM}, \mathrm{RI} / \mathrm{SmOM}_{\gamma_{\mu}}\right\}$. The anomalous dimensions 
can again be obtained to three-loop order in the Landau gauge by inserting the result for the matching factors $C_{T, L}^{x}$ of Eqs. (23) and (24) into

$$
\gamma_{T}^{x}=\gamma_{T}^{\overline{\mathrm{MS}}}-\beta \frac{\partial \log C_{T, L}^{x}}{\partial \frac{\alpha_{s}}{4 \pi}}, \quad x \in\left\{\mathrm{RI} / \mathrm{SMOM}, \mathrm{RI} / \mathrm{SMOM}_{\gamma_{\mu}}\right\} .
$$

The $\overline{\mathrm{MS}}$ anomalous dimension $\gamma_{T}^{\overline{\mathrm{MS}}}$ of the tensor operator is known up to three-loop order and can be taken from Refs. [39, 40, 9]. It is also given explicitly in Appendix C. The results for the RI/SMOM and RI/SMOM ${ }_{\gamma_{\mu}}$ schemes read

$$
\begin{aligned}
& -\gamma_{T}^{(2), \mathrm{RI} / \mathrm{SMOM}}=C_{F}^{3}\left[-\frac{365}{6}+64 \zeta_{3}\right]+C_{A}^{2} C_{F}\left[-\frac{69607}{162}-\frac{7693}{162} \Psi^{\prime}\left(\frac{1}{3}\right)\right. \\
& +\frac{44}{81} \Psi^{\prime}\left(\frac{1}{3}\right)^{2}+\frac{7693}{243} \pi^{2}-\frac{176}{243} \Psi^{\prime}\left(\frac{1}{3}\right) \pi^{2}+\frac{770}{729} \pi^{4}-\frac{11}{36} \Psi^{\prime \prime \prime}\left(\frac{1}{3}\right)+\frac{2816}{3} s_{2}\left(\frac{\pi}{2}\right) \\
& -\frac{1408}{3} s_{2}\left(\frac{\pi}{6}\right)-\frac{5632}{9} s_{3}\left(\frac{\pi}{2}\right)+\frac{7040}{9} s_{3}\left(\frac{\pi}{6}\right)+\frac{55}{9} \Sigma+\frac{352 \log (3) \pi}{9 \sqrt{3}} \\
& \left.-\frac{88 \log ^{2}(3) \pi}{27 \sqrt{3}}+\frac{2552 \pi^{3}}{729 \sqrt{3}}+\frac{1460}{9} \zeta_{3}\right] \\
& +C_{A} C_{F}^{2}\left[\frac{9883}{27}+\frac{4004}{27} \Psi^{\prime}\left(\frac{1}{3}\right)-\frac{121}{81} \Psi^{\prime}\left(\frac{1}{3}\right)^{2}-\frac{8008}{81} \pi^{2}+\frac{484}{243} \Psi^{\prime}\left(\frac{1}{3}\right) \pi^{2}\right. \\
& +\frac{572}{729} \pi^{4}-\frac{44}{81} \Psi^{\prime \prime \prime}\left(\frac{1}{3}\right)-1760 s_{2}\left(\frac{\pi}{2}\right)+880 s_{2}\left(\frac{\pi}{6}\right)+\frac{3520}{3} s_{3}\left(\frac{\pi}{2}\right) \\
& \left.-\frac{4400}{3} s_{3}\left(\frac{\pi}{6}\right)+\frac{22}{3} \Sigma-\frac{220 \log (3) \pi}{3 \sqrt{3}}+\frac{55 \log ^{2}(3) \pi}{9 \sqrt{3}}-\frac{1595 \pi^{3}}{243 \sqrt{3}}-200 \zeta_{3}\right] \\
& +n_{f} T_{F}\left\{C _ { A } C _ { F } \left[\frac{17426}{81}+\frac{890}{81} \Psi^{\prime}\left(\frac{1}{3}\right)-\frac{16}{81} \Psi^{\prime}\left(\frac{1}{3}\right)^{2}-\frac{1780}{243} \pi^{2}+\frac{64}{243} \Psi^{\prime}\left(\frac{1}{3}\right) \pi^{2}\right.\right. \\
& -\frac{280}{729} \pi^{4}+\frac{1}{9} \Psi^{\prime \prime \prime}\left(\frac{1}{3}\right)-\frac{1024}{3} s_{2}\left(\frac{\pi}{2}\right)+\frac{512}{3} s_{2}\left(\frac{\pi}{6}\right)+\frac{2048}{9} s_{3}\left(\frac{\pi}{2}\right)-\frac{2560}{9} s_{3}\left(\frac{\pi}{6}\right) \\
& \left.-\frac{20}{9} \Sigma-\frac{128 \log (3) \pi}{9 \sqrt{3}}+\frac{32 \log ^{2}(3) \pi}{27 \sqrt{3}}-\frac{928 \pi^{3}}{729 \sqrt{3}}-\frac{256}{9} \zeta_{3}\right] \\
& +C_{F}^{2}\left[-\frac{1883}{27}-\frac{1492}{27} \Psi^{\prime}\left(\frac{1}{3}\right)+\frac{44}{81} \Psi^{\prime}\left(\frac{1}{3}\right)^{2}+\frac{2984}{81} \pi^{2}-\frac{176}{243} \Psi^{\prime}\left(\frac{1}{3}\right) \pi^{2}-\frac{208}{729} \pi^{4}\right. \\
& +\frac{16}{81} \Psi^{\prime \prime \prime}\left(\frac{1}{3}\right)+640 s_{2}\left(\frac{\pi}{2}\right)-320 s_{2}\left(\frac{\pi}{6}\right)-\frac{1280}{3} s_{3}\left(\frac{\pi}{2}\right)+\frac{1600}{3} s_{3}\left(\frac{\pi}{6}\right)-\frac{8}{3} \Sigma \\
& \left.\left.+\frac{80 \log (3) \pi}{3 \sqrt{3}}-\frac{20 \log ^{2}(3) \pi}{9 \sqrt{3}}+\frac{580 \pi^{3}}{243 \sqrt{3}}+16 \zeta_{3}\right]\right\} \\
& +C_{F} n_{f}^{2} T_{F}^{2}\left[-\frac{1784}{81}+\frac{160}{81} \Psi^{\prime}\left(\frac{1}{3}\right)-\frac{320}{243} \pi^{2}\right]
\end{aligned}
$$

and

$$
-\gamma_{T}^{(2), \mathrm{RI} / \mathrm{SMOM}_{\gamma_{\mu}}}=C_{F}^{3}\left[-\frac{365}{6}+64 \zeta_{3}\right]+C_{A}^{2} C_{F}\left[-\frac{112211}{324}-\frac{1817}{81} \Psi^{\prime}\left(\frac{1}{3}\right)\right.
$$




$$
\begin{aligned}
& -\frac{22}{81} \Psi^{\prime}\left(\frac{1}{3}\right)^{2}+\frac{3634}{243} \pi^{2}+\frac{88}{243} \Psi^{\prime}\left(\frac{1}{3}\right) \pi^{2}+\frac{308}{729} \pi^{4}-\frac{11}{54} \Psi^{\prime \prime \prime}\left(\frac{1}{3}\right)+\frac{2156}{3} s_{2}\left(\frac{\pi}{2}\right) \\
& -\frac{1078}{3} s_{2}\left(\frac{\pi}{6}\right)-\frac{4312}{9} s_{3}\left(\frac{\pi}{2}\right)+\frac{5390}{9} s_{3}\left(\frac{\pi}{6}\right)+\frac{55}{9} \Sigma+\frac{539 \log (3) \pi}{18 \sqrt{3}} \\
& \left.-\frac{539 \log ^{2}(3) \pi}{216 \sqrt{3}}+\frac{15631 \pi^{3}}{5832 \sqrt{3}}+\frac{1196}{9} \zeta_{3}\right] \\
+ & C_{A} C_{F}^{2}\left[\frac{19271}{54}+\frac{2717}{27} \Psi^{\prime}\left(\frac{1}{3}\right)+\frac{11}{81} \Psi^{\prime}\left(\frac{1}{3}\right)^{2}-\frac{5434}{81} \pi^{2}-\frac{44}{243} \Psi^{\prime}\left(\frac{1}{3}\right) \pi^{2}+\frac{704}{729} \pi^{4}\right. \\
& -\frac{55}{162} \Psi^{\prime \prime \prime}\left(\frac{1}{3}\right)-1408 s_{2}\left(\frac{\pi}{2}\right)+704 s_{2}\left(\frac{\pi}{6}\right)+\frac{2816}{3} s_{3}\left(\frac{\pi}{2}\right)-\frac{3520}{3} s_{3}\left(\frac{\pi}{6}\right)+\frac{22}{3} \Sigma \\
& \left.-\frac{176 \log (3) \pi}{3 \sqrt{3}}+\frac{44 \log ^{2}(3) \pi}{9 \sqrt{3}}-\frac{1276 \pi^{3}}{243 \sqrt{3}}-\frac{644}{3} \zeta_{3}\right] \\
+ & n_{f} T_{F}\left\{C _ { A } C _ { F } \left[\frac{12851}{81}+\frac{152}{81} \Psi^{\prime}\left(\frac{1}{3}\right)+\frac{8}{81} \Psi^{\prime}\left(\frac{1}{3}\right)^{2}-\frac{304}{243} \pi^{2}-\frac{32}{243} \Psi^{\prime}\left(\frac{1}{3}\right) \pi^{2}\right.\right. \\
& -\frac{112}{729} \pi^{4}+\frac{2}{27} \Psi^{\prime \prime \prime}\left(\frac{1}{3}\right)-\frac{784}{3} s_{2}\left(\frac{\pi}{2}\right)+\frac{392}{3} s_{2}\left(\frac{\pi}{6}\right)+\frac{1568}{9} s_{3}\left(\frac{\pi}{2}\right)-\frac{1960}{9} s_{3}\left(\frac{\pi}{6}\right) \\
& \left.-\frac{20}{9} \Sigma-\frac{98 \log (3) \pi}{9 \sqrt{3}}+\frac{49 \log ^{2}(3) \pi}{54 \sqrt{3}}-\frac{1421 \pi^{3}}{1458 \sqrt{3}}-\frac{160}{9} \zeta_{3}\right] \\
+ & C_{F}^{2}\left[-\frac{1901}{27}-\frac{1024}{27} \Psi_{f}^{2} T_{F}^{2}\left[\frac{1}{3}\right)-\frac{4}{81} \Psi^{\prime}\left(\frac{1}{3}\right)^{2}+\frac{2048}{81} \pi^{2}+\frac{16}{243} \Psi^{\prime}\left(\frac{1}{3}\right) \pi^{2}-\frac{256}{729} \pi^{4}\right. \\
& +\frac{10}{81} \Psi^{\prime \prime \prime}\left(\frac{1}{3}\right)+512 s_{2}\left(\frac{\pi}{2}\right)-256 s_{2}\left(\frac{\pi}{6}\right)-\frac{1024}{3} s_{3}\left(\frac{\pi}{2}\right)+\frac{1280}{3} s_{3}\left(\frac{\pi}{6}\right) \\
& \left.\left.-\frac{8}{3} \Sigma+\frac{64 \log (3) \pi}{3 \sqrt{3}}-\frac{16 \log ^{2}(3) \pi}{9 \sqrt{3}}+\frac{464 \pi^{3}}{243 \sqrt{3}}+\frac{64}{3} \zeta_{3}\right]\right\} \\
& {\left[31\left(\frac{1}{3}\right)-\frac{320}{243} \pi^{2}\right] \cdot }
\end{aligned}
$$

\section{Summary and Conclusion}

We have computed the two-loop QCD corrections for matching factors which convert light quark masses renormalized in the RI/SMOM and RI/SMOM $\gamma_{\mu}$ schemes to the $\overline{\mathrm{MS}}$ scheme. These schemes are extensions of the traditional regularization independent momentumsubtraction schemes, like the RI/MOM or $\mathrm{RI}^{\prime}$ /MOM scheme. The latter are characterized by an exceptional subtraction point, whereas in the RI/SMOM and RI/SMOM $\gamma_{\mu}$ schemes the ultraviolet divergences are subtracted at a symmetric subtraction point. This allows for a lattice simulation with suppressed contamination from infrared effects. The perturbative expansion coefficients at two-loop order are significantly smaller than in the traditional RI/MOM and $\mathrm{RI}^{\prime} / \mathrm{MOM}$ schemes, with about $0.6 \%-0.7 \%$ and $2 \%$ of the leading order result for the RI/SMOM and RI/SMOM $\gamma_{\mu}$ schemes for $n_{f}=3$ flavors at scales of about $2 \mathrm{GeV}$. We also provide the results for the three-loop anomalous dimensions of the quark field and the mass in both schemes. The mass anomalous dimension can be 
used to run the quark masses to different energy scales.

The use of these matching factors will reduce the uncertainties associated with the matching procedure for converting the quark mass from the regularization invariant momentum-subtraction scheme to the $\overline{\mathrm{MS}}$ scheme due to smaller truncation errors of the perturbative series. These results will allow for an $\overline{\mathrm{MS}}$ light quark mass determination only together with lattice simulations in the context of nonperturbative renormalization, with a significant reduced systematic error compared to previous determinations which use momentum-subtraction schemes with an exceptional subtraction point, whose matching factors show a poor convergence behavior at the required energy scales.

In addition, the availability of both the RI/SMOM and RI/SMOM $\gamma_{\mu}$ schemes at twoloop order will also allow for a better assessment and control of the uncertainties. This can be achieved by converting results derived in the different regularization invariant momentum-subtraction schemes to the $\overline{\mathrm{MS}}$ scheme, using the above matching factors, and subsequently comparing and cross-checking the obtained $\overline{\mathrm{MS}}$ results.

Our computation is accomplished by studying at two-loop order quark bilinear operators inserted into amputated Green's functions for the vector, axial-vector, scalar, and pseudoscalar operators. We also provide the corresponding results for the matching factors and the anomalous dimensions of the tensor operator.

\section{Acknowledgments:}

We are grateful to our colleagues of the RBC-UKQCD Collaborations for many valuable discussions, in particular, to A. Soni for advice and encouragement. We would like to thank T. Izubuchi and S. Uccirati for conversations about the master integrals as well as Y. Aoki for important discussions. This work was supported by the U.S. DOE under Contract No. DE-AC02-98CH10886.

\section{Note added:}

During the finalization of this paper Ref. [41] appeared, where the two-loop QCD corrections of the matching factor $C_{m}^{\mathrm{RI} / \mathrm{SMOM}}$ have also been determined. The result in Eq. (29) of Ref. [41] is for the symmetric subtraction point in agreement with our result in Eq. (21). In the revised version the authors of Ref. [41] provide additional results which confirm our

results for $C_{q}^{\mathrm{RI} / \mathrm{SMOM}_{\gamma \mu}}$ and $C_{m}^{\mathrm{RI} / \mathrm{SMOM}_{\gamma \mu}}$; the corresponding anomalous dimensions agree up to a global sign which has been rectified in the current version. We would like to thank the authors of Ref. [41] for communications and for pointing this out to us.

\section{A Master integrals and harmonic polylogarithms}

The harmonic polylogarithms $H$ of Ref. 42] are defined recursively as

$$
H\left(a, a_{1}, \ldots, a_{k} ; x\right)=\int_{0}^{x} d x^{\prime} f_{a}\left(x^{\prime}\right) H\left(a_{1}, \ldots, a_{k} ; x^{\prime}\right)
$$

with

$$
f_{1}(x)=\frac{1}{1-x}, \quad f_{0}(x)=\frac{1}{x}, \quad f_{-1}(x)=\frac{1}{1+x}
$$


and

$$
H(1 ; x)=-\log (1-x), \quad H(0 ; x)=\log (x), \quad H(-1 ; x)=\log (1+x) .
$$

The constants $\mathcal{H}_{31}^{(2)}$ and $\mathcal{H}_{43}^{(2)}$ in the master integrals of Eqs. (11) and (16) can be expressed in terms of harmonic polylogarithm, 4 ; their numerical evaluation is given by

$$
\mathcal{H}_{31}^{(2)}=-6.11477558 \ldots, \quad \mathcal{H}_{43}^{(2)}=+12.45994893 \ldots
$$

In the conversion factors these constants always arise in the form of the sum $\Sigma=\mathcal{H}_{31}^{(2)}+$ $\mathcal{H}_{43}^{(2)}$ in which some of the harmonic polylogarithms cancel. It can be expressed with $z_{0}=\frac{i}{\sqrt{3}}$ by

$$
\begin{aligned}
\Sigma= & \frac{1}{3 \sqrt{3}}\left[12 \pi \zeta_{3}-12 \pi H\left(0,+,+; z_{0}\right)-6 \pi H\left(-,+,+; z_{0}\right)+i \pi^{2} H\left(-,+; z_{0}\right)\right. \\
& \left.+2 i \pi^{2} H\left(0,+; z_{0}\right)-18 i H\left(-,+,+,+; z_{0}\right)-36 i H\left(0,+,+,+; z_{0}\right)\right] \\
\simeq & 6.34517334592058543866471875422848044360,
\end{aligned}
$$

where the functions $H( \pm, \cdots, \pm ; x)$ are defined by the following linear combinations:

$$
\begin{aligned}
H(+; x) & =H(1 ; x)+H(-1 ; x) \\
H(-; x) & =H(1 ; x)-H(-1 ; x) \\
H\left( \pm, a_{1}, \ldots, a_{k} ; x\right) & =H\left(1, a_{1}, \ldots, a_{k} ; x\right) \pm H\left(-1, a_{1}, \ldots, a_{k} ; x\right) .
\end{aligned}
$$

\section{B Gauge dependent parts of the conversion factors}

In this section we provide the gauge dependent components of the conversion factors, where the gauge parameter $\xi$ has been renormalized in the $\overline{\mathrm{MS}}$ scheme. As mentioned in Section 4.1, the fermion field conversion factors in the RI/SMOM scheme are given by the ones in the $\mathrm{RI}^{\prime} / \mathrm{MOM}$ scheme; therefore we omit them here. However, we did check that the gauge dependent terms were also in agreement with the previous calculations in the $\mathrm{RI}^{\prime} / \mathrm{MOM}$ scheme. The results read as follows:

$$
\begin{aligned}
C_{q, \xi}^{\mathrm{RI} / \mathrm{SMOM}_{\gamma_{\mu}}}= & \left(\frac{\alpha_{s}}{4 \pi}\right) C_{F} \xi\left[\frac{1}{3} \Psi^{\prime}\left(\frac{1}{3}\right)-\frac{3}{2}-\frac{2 \pi^{2}}{9}\right] \\
+ & \left(\frac{\alpha_{s}}{4 \pi}\right)^{2}\left\{C _ { F } ^ { 2 } \left[\xi \left(\Sigma-1+2 \zeta_{3}-\frac{31 \pi^{2}}{9}-\frac{29 \pi^{3}}{162 \sqrt{3}}+\frac{4 \pi^{4}}{27}\right.\right.\right. \\
& +24 s_{2}\left(\frac{\pi}{6}\right)-48 s_{2}\left(\frac{\pi}{2}\right)-40 s_{3}\left(\frac{\pi}{6}\right)+32 s_{3}\left(\frac{\pi}{2}\right)-\frac{2 \pi \log (3)}{\sqrt{3}}
\end{aligned}
$$

\footnotetext{
${ }^{4}$ The $\varepsilon$ expansion of the master integral $\mathcal{M}_{31}^{(1)}$ can also be obtained with Refs. 43, 44].

${ }^{5}$ We have checked that our numerical evaluation using traditional Feynman parametrizations of these constants agrees with the numerically evaluated analytical result.
} 


$$
\begin{aligned}
& \left.+\frac{\pi \log ^{2}(3)}{6 \sqrt{3}}+\frac{31}{6} \Psi^{\prime}\left(\frac{1}{3}\right)-\frac{1}{18} \Psi^{\prime \prime \prime}\left(\frac{1}{3}\right)\right)+\xi^{2}\left(\frac{7}{4}+\frac{4 \pi^{2}}{9}+\frac{4 \pi^{4}}{81}\right. \\
& \left.\left.-\frac{2}{3} \Psi^{\prime}\left(\frac{1}{3}\right)-\frac{4 \pi^{2}}{27} \Psi^{\prime}\left(\frac{1}{3}\right)+\frac{1}{9} \Psi^{\prime}\left(\frac{1}{3}\right)^{2}\right)\right] \\
& +C_{A} C_{F}\left[\xi \left(-\frac{33}{4}+\frac{2 \pi^{2}}{9}+\frac{29 \pi^{3}}{432 \sqrt{3}}+\frac{\pi^{4}}{27}+\frac{1}{2} \Sigma+3 \zeta_{3}+\frac{\sqrt{3} \pi \log (3)}{4}\right.\right. \\
& -\frac{1}{3} \Psi^{\prime}\left(\frac{1}{3}\right)-\frac{1}{72} \Psi^{\prime \prime \prime}\left(\frac{1}{3}\right)-9 s_{2}\left(\frac{\pi}{6}\right)+18 s_{2}\left(\frac{\pi}{2}\right)-12 s_{3}\left(\frac{\pi}{2}\right)+15 s_{3}\left(\frac{\pi}{6}\right) \\
& \left.\left.\left.-\frac{\pi \log ^{2}(3)}{16 \sqrt{3}}\right)+\xi^{2}\left(\frac{1}{4} \Psi^{\prime}\left(\frac{1}{3}\right)-\frac{3}{2}-\frac{\pi^{2}}{6}\right)\right]\right\}+\mathcal{O}\left(\alpha_{s}^{3}\right) \\
& C_{m, \xi}^{\mathrm{RI} / \mathrm{SMOM}}=\left(\frac{\alpha_{s}}{4 \pi}\right) C_{F} \xi\left(\frac{1}{3} \Psi^{\prime}\left(\frac{1}{3}\right)-1-\frac{2 \pi^{2}}{9}\right) \\
& +\left(\frac{\alpha_{s}}{4 \pi}\right)^{2}\left\{C _ { F } ^ { 2 } \left[\xi \left(4+\frac{8 \pi^{2}}{9}+\frac{4 \pi^{4}}{9}+\Sigma-\frac{4}{3} \Psi^{\prime}\left(\frac{1}{3}\right)-\frac{8 \pi^{2}}{9} \Psi^{\prime}\left(\frac{1}{3}\right)+\frac{2}{3} \Psi^{\prime}\left(\frac{1}{3}\right)^{2}\right.\right.\right. \\
& \left.\left.-\frac{1}{18} \Psi^{\prime \prime \prime}\left(\frac{1}{3}\right)\right)+\xi^{2}\left(1+\frac{4 \pi^{2}}{9}+\frac{4 \pi^{4}}{81}-\frac{2}{3} \Psi^{\prime}\left(\frac{1}{3}\right)-\frac{4 \pi^{2}}{27} \Psi^{\prime}\left(\frac{1}{3}\right)+\frac{1}{9} \Psi^{\prime}\left(\frac{1}{3}\right)^{2}\right)\right] \\
& +C_{A} C_{F}\left[\xi\left(\frac{7}{6} \Psi^{\prime}\left(\frac{1}{3}\right)-\frac{7}{2}-\frac{7 \pi^{2}}{9}+\frac{\pi^{4}}{27}+\frac{\Sigma}{2}-\frac{1}{72} \Psi^{\prime \prime \prime}\left(\frac{1}{3}\right)\right)\right. \\
& \left.\left.+\xi^{2}\left(\frac{1}{4} \Psi^{\prime}\left(\frac{1}{3}\right)-\frac{3}{4}-\frac{\pi^{2}}{6}\right)\right]\right\}+\mathcal{O}\left(\alpha_{s}^{3}\right) \\
& C_{m, \xi}^{\mathrm{RI} / \mathrm{SMOM}_{\gamma \mu}}=-\left(\frac{\alpha_{s}}{4 \pi}\right) \frac{1}{2} C_{F} \xi \\
& +\left(\frac{\alpha_{s}}{4 \pi}\right)^{2}\left\{C _ { F } ^ { 2 } \left[\xi \left(2+\frac{26 \pi^{2}}{9}+\frac{29 \pi^{3}}{162 \sqrt{3}}+\frac{4 \pi^{4}}{27}-2 \zeta_{3}+\frac{2 \pi \log (3)}{\sqrt{3}}\right.\right.\right. \\
& -24 s_{2}\left(\frac{\pi}{6}\right)+48 s_{2}\left(\frac{\pi}{2}\right)+40 s_{3}\left(\frac{\pi}{6}\right)-32 s_{3}\left(\frac{\pi}{2}\right)-\frac{\pi \log ^{2}(3)}{6 \sqrt{3}} \\
& \left.\left.-\frac{13}{3} \Psi^{\prime}\left(\frac{1}{3}\right)-\frac{4 \pi^{2}}{9} \Psi^{\prime}\left(\frac{1}{3}\right)+\frac{1}{3} \Psi^{\prime}\left(\frac{1}{3}\right)^{2}\right)+\xi^{2}\left(\frac{1}{2}+\frac{\pi^{2}}{9}-\frac{1}{6} \Psi^{\prime}\left(\frac{1}{3}\right)\right)\right] \\
& +C_{A} C_{F}\left[-\frac{3}{8} \xi^{2}+\xi\left(-\frac{7}{4}-\pi^{2}-\frac{29 \pi^{3}}{432 \sqrt{3}}+9 s_{2}\left(\frac{\pi}{6}\right)-18 s_{2}\left(\frac{\pi}{2}\right)-15 s_{3}\left(\frac{\pi}{6}\right)\right.\right. \\
& \left.\left.\left.+12 s_{3}\left(\frac{\pi}{2}\right)-\frac{\sqrt{3} \pi \log (3)}{4}+\frac{\pi \log ^{2}(3)}{16 \sqrt{3}}+\frac{3}{2} \Psi^{\prime}\left(\frac{1}{3}\right)\right)\right]\right\}+\mathcal{O}\left(\alpha_{s}^{3}\right),
\end{aligned}
$$




$$
\begin{aligned}
& +\left(\frac{\alpha_{s}}{4 \pi}\right)^{2}\left\{C _ { F } ^ { 2 } \xi \left[-6 \Psi^{\prime}\left(\frac{1}{3}\right)+4 \pi^{2}-\frac{4}{27} \pi^{4}+\frac{1}{18} \Psi^{\prime \prime \prime}\left(\frac{1}{3}\right)+64 s_{2}\left(\frac{\pi}{2}\right)-32 s_{2}\left(\frac{\pi}{6}\right)\right.\right. \\
& \left.-\frac{128}{3} s_{3}\left(\frac{\pi}{2}\right)+\frac{160}{3} s_{3}\left(\frac{\pi}{6}\right)-\Sigma+\frac{8 \log (3) \pi}{3 \sqrt{3}}-\frac{2 \log ^{2}(3) \pi}{9 \sqrt{3}}+\frac{58 \pi^{3}}{243 \sqrt{3}}-\frac{8}{3} \zeta_{3}\right] \\
& +C_{A} C_{F}\left[\xi \left(\frac{7}{6}+\frac{5}{6} \Psi^{\prime}\left(\frac{1}{3}\right)-\frac{5}{9} \pi^{2}-\frac{1}{27} \pi^{4}+\frac{1}{72} \Psi^{\prime \prime \prime}\left(\frac{1}{3}\right)-24 s_{2}\left(\frac{\pi}{2}\right)\right.\right. \\
& \left.+12 s_{2}\left(\frac{\pi}{6}\right)+16 s_{3}\left(\frac{\pi}{2}\right)-20 s_{3}\left(\frac{\pi}{6}\right)-\frac{1}{2} \Sigma-\frac{\log (3) \pi}{\sqrt{3}}+\frac{\log ^{2}(3) \pi}{12 \sqrt{3}}-\frac{29 \pi^{3}}{324 \sqrt{3}}\right) \\
& \left.\left.+\xi^{2}\left(\frac{1}{4}-\frac{1}{4} \Psi^{\prime}\left(\frac{1}{3}\right)+\frac{1}{6} \pi^{2}\right)\right]\right\}+\mathcal{O}\left(\alpha_{s}^{3}\right) \\
& C_{T, \xi}^{\mathrm{RI} / \mathrm{SMOM}_{\gamma \mu}}=-\left(\frac{\alpha_{s}}{4 \pi}\right) \frac{1}{6} C_{F} \xi \\
& +\left(\frac{\alpha_{s}}{4 \pi}\right)^{2}\left\{C _ { F } ^ { 2 } \left[\xi \left(1-\frac{16}{9} \Psi^{\prime}\left(\frac{1}{3}\right)+\frac{1}{9} \Psi^{\prime}\left(\frac{1}{3}\right)^{2}+\frac{32}{27} \pi^{2}-\frac{4}{27} \Psi^{\prime}\left(\frac{1}{3}\right) \pi^{2}\right.\right.\right. \\
& +\frac{4}{81} \pi^{4}+16 s_{2}\left(\frac{\pi}{2}\right)-8 s_{2}\left(\frac{\pi}{6}\right)-\frac{32}{3} s_{3}\left(\frac{\pi}{2}\right)+\frac{40}{3} s_{3}\left(\frac{\pi}{6}\right)+\frac{2 \log (3) \pi}{3 \sqrt{3}} \\
& \left.\left.-\frac{\log ^{2}(3) \pi}{18 \sqrt{3}}+\frac{29 \pi^{3}}{486 \sqrt{3}}-\frac{2}{3} \zeta_{3}\right)+\xi^{2}\left(\frac{1}{12}-\frac{1}{18} \Psi^{\prime}\left(\frac{1}{3}\right)+\frac{1}{27} \pi^{2}\right)\right] \\
& +C_{A} C_{F}\left[-\frac{1}{8} \xi^{2}+\xi\left(-\frac{7}{12}+\frac{1}{2} \Psi^{\prime}\left(\frac{1}{3}\right)-\frac{1}{3} \pi^{2}-6 s_{2}\left(\frac{\pi}{2}\right)+3 s_{2}\left(\frac{\pi}{6}\right)+4 s_{3}\left(\frac{\pi}{2}\right)\right.\right. \\
& \left.\left.\left.-5 s_{3}\left(\frac{\pi}{6}\right)-\frac{\log (3) \pi}{4 \sqrt{3}}+\frac{\log ^{2}(3) \pi}{48 \sqrt{3}}-\frac{29 \pi^{3}}{1296 \sqrt{3}}\right)\right]\right\}+\mathcal{O}\left(\alpha_{s}^{3}\right) .
\end{aligned}
$$

\section{$\mathrm{C}$ The QCD $\beta$ function and anomalous dimensions in the $\overline{\mathrm{MS}}$ scheme}

The QCD $\beta$ function is defined by

$$
\beta=\frac{d \frac{\alpha_{s}}{4 \pi}}{d \log \mu^{2}}=-\beta_{0}\left(\frac{\alpha_{s}}{4 \pi}\right)^{2}-\beta_{1}\left(\frac{\alpha_{s}}{4 \pi}\right)^{3}+\mathcal{O}\left(\alpha_{s}^{4}\right)
$$

and known up to four-loop order [45,54]. The lowest two orders are given by

$$
\begin{aligned}
& \beta_{0}=\frac{11}{3} C_{A}-\frac{4}{3} T_{F} n_{f}, \\
& \beta_{1}=\frac{34}{3} C_{A}^{2}-4 C_{F} T_{F} n_{f}-\frac{20}{3} C_{A} T_{F} n_{f} .
\end{aligned}
$$

The $\overline{\mathrm{MS}}$ mass anomalous dimension [55, 56, 27, 57, 58] is defined in Eq. (29) and reads up to order $\alpha_{s}^{3}$

$$
\gamma_{m}^{(0), \overline{\mathrm{MS}}}=3 C_{F}
$$




$$
\begin{aligned}
\gamma_{m}^{(1), \overline{\mathrm{MS}}}= & \frac{3}{2} C_{F}^{2}+\frac{97}{6} C_{F} C_{A}-\frac{10}{3} C_{F} T_{F} n_{f}, \\
\gamma_{m}^{(2), \overline{\mathrm{MS}}=} & \frac{129}{2} C_{F}^{3}-\frac{129}{4} C_{F}^{2} C_{A}+\frac{11413}{108} C_{F} C_{A}^{2}+C_{F}^{2} T_{F} n_{f}\left(48 \zeta_{3}-46\right) \\
& -C_{F} C_{A} T_{F} n_{f}\left(48 \zeta_{3}+\frac{556}{27}\right)-\frac{140}{27} C_{F} T_{F}^{2} n_{f}^{2} .
\end{aligned}
$$

The lowest orders of the $\overline{\mathrm{MS}}$ anomalous dimension of the fermion field [8, 9] defined in Eq. (33) for $\xi=0$ (Landau gauge) are given by

$$
\begin{aligned}
\gamma_{q}^{(0), \overline{\mathrm{MS}}}= & 0, \quad \gamma_{q}^{(1), \overline{\mathrm{MS}}}=-C_{F}^{2} \frac{3}{2}+C_{F} C_{A} \frac{25}{4}-2 C_{F} T_{F} n_{f}, \\
\gamma_{q}^{(2), \overline{\mathrm{MS}}}= & C_{F}^{3} \frac{3}{2}-C_{A} C_{F}^{2}\left(\frac{143}{4}-12 \zeta_{3}\right)+C_{A}^{2} C_{F}\left(\frac{9155}{144}-\frac{69}{8} \zeta_{3}\right) \\
& +n_{f} T_{F}\left(3 C_{F}^{2}-C_{A} C_{F} \frac{287}{9}\right)+C_{F} n_{f}^{2} T_{F}^{2} \frac{20}{9} .
\end{aligned}
$$

The Casimir operators in Eqs. (52)-(58) of the $\mathrm{SU}\left(N_{c}\right)$ group are given by $C_{A}=N_{c}$, $C_{F}=\left(N_{c}^{2}-1\right) /\left(2 N_{c}\right)$, where $N_{c}$ is the number of colors.

For our calculation of the tensor anomalous dimensions in the RI/SMOM and RI/SMOM $\gamma_{\mu}$ schemes, we need the corresponding $\overline{\mathrm{MS}}$ anomalous dimension of the tensor operator up to three-loop order [39, 40, 9], which we have taken from these references. The first three orders read

$$
\begin{aligned}
\gamma_{T}^{(0), \overline{\mathrm{MS}}}= & C_{F}, \quad \gamma_{T}^{(1), \overline{\mathrm{MS}}}=-C_{F}^{2} \frac{19}{2}+C_{F} C_{A} \frac{257}{18}-C_{F} n_{f} T_{F} \frac{26}{9} \\
\gamma_{T}^{(2), \overline{\mathrm{MS}}}= & -C_{F}^{3}\left(64 \zeta_{3}-\frac{365}{6}\right)+C_{A} C_{F}^{2}\left(112 \zeta_{3}-\frac{6823}{36}\right)-C_{A}^{2} C_{F}\left(40 \zeta_{3}-\frac{13639}{108}\right) \\
& +n_{f} T_{F}\left[C_{F}^{2}\left(16 \zeta_{3}+\frac{98}{9}\right)-C_{A} C_{F}\left(16 \zeta_{3}+\frac{1004}{27}\right)\right]-\frac{4}{3} C_{F} n_{f}^{2} T_{F}^{2}
\end{aligned}
$$

\section{The one- and two-loop anomalous dimensions in the RI/SMOM$\left(\gamma_{\mu}\right)$ scheme}

The one- and two-loop anomalous dimensions have already been determined in Ref. [11]. In order to avoid conventional subtleties we also provide these results here for completeness. The one-loop results in the Landau gauge are given by

$$
\begin{aligned}
\gamma_{m}^{(0), \mathrm{RI} / \mathrm{SMOM}} & =3 C_{F}=\gamma_{m}^{(0), \mathrm{RI} / \mathrm{SMOM}_{\gamma \mu}}, \quad \gamma_{q}^{(0), \mathrm{RI} / \mathrm{SMOM}_{\gamma_{\mu}}}=0 \\
\gamma_{T}^{(0), \mathrm{RI} / \mathrm{SMOM}} & =C_{F}=\gamma_{T}^{(0), \mathrm{RI} / \mathrm{SMOM}_{\gamma_{\mu}}} .
\end{aligned}
$$

The two-loop results in the Landau gauge read

$$
-\gamma_{m}^{(1), \mathrm{RI} / \mathrm{SMOM}}=-C_{F}^{2} \frac{3}{2}-C_{A} C_{F}\left(\frac{185}{6}+\frac{22}{9} \pi^{2}-\frac{11}{3} \Psi^{\prime}\left(\frac{1}{3}\right)\right)
$$




$$
\begin{aligned}
& -C_{F} T_{F} n_{f}\left(\frac{4}{3} \Psi^{\prime}\left(\frac{1}{3}\right)-\frac{26}{3}-\frac{8}{9} \pi^{2}\right) \\
& -\gamma_{m}^{(1), \mathrm{RI} / \mathrm{SMOM}_{\gamma_{\mu}}}=-C_{F}^{2} \frac{3}{2}-C_{A} C_{F}\left(\frac{69}{2}+\frac{22}{9} \pi^{2}-\frac{11}{3} \Psi^{\prime}\left(\frac{1}{3}\right)\right) \\
& \text { - } C_{F} T_{F} n_{f}\left(\frac{4}{3} \Psi^{\prime}\left(\frac{1}{3}\right)-10-\frac{8}{9} \pi^{2}\right) \text {, } \\
& -\gamma_{q}^{(1), \mathrm{RI} / \mathrm{SMOM}_{\gamma \mu}}=C_{F}^{2} \frac{3}{2}-C_{A} C_{F} \frac{31}{12}+C_{F} T_{F} n_{f} \frac{2}{3}, \\
& -\gamma_{T}^{(1), \mathrm{RI} / \mathrm{SMOM}}=C_{F}^{2} \frac{19}{2}-C_{A} C_{F}\left(\frac{115}{6}-\frac{11}{9} \Psi^{\prime}\left(\frac{1}{3}\right)+\frac{22}{27} \pi^{2}\right) \\
& +C_{F} n_{f} T_{F}\left(\frac{14}{3}-\frac{4}{9} \Psi^{\prime}\left(\frac{1}{3}\right)+\frac{8}{27} \pi^{2}\right) \text {, } \\
& -\gamma_{T}^{(1), \mathrm{RI} / \mathrm{SMOM}_{\gamma_{\mu}}}=C_{F}^{2} \frac{19}{2}-C_{A} C_{F}\left(\frac{31}{2}-\frac{11}{9} \Psi^{\prime}\left(\frac{1}{3}\right)+\frac{22}{27} \pi^{2}\right) \\
& +C_{F} n_{f} T_{F}\left(\frac{10}{3}-\frac{4}{9} \Psi^{\prime}\left(\frac{1}{3}\right)+\frac{8}{27} \pi^{2}\right) \text {. }
\end{aligned}
$$

\section{References}

[1] G. Martinelli, C. Pittori, C. T. Sachrajda, M. Testa and A. Vladikas, Nucl. Phys. B445 (1995) 81.

[2] Y. Aoki, PoS LAT2009 (2009) 012.

[3] C. Allton et al., Phys. Rev. D78 (2008) 114509.

[4] G. 't Hooft, Nucl. Phys. B61 (1973) 455.

[5] W. A. Bardeen, A. J. Buras, D. W. Duke and T. Muta, Phys. Rev. D18 (1978) 3998.

[6] G. 't Hooft and M. J. G. Veltman, Nucl. Phys. B44 (1972) 189.

[7] E. Franco and V. Lubicz, Nucl. Phys. B531 (1998) 641.

[8] K. G. Chetyrkin and A. Retey, Nucl. Phys. B583 (2000) 3.

[9] J. A. Gracey, Nucl. Phys. B662 (2003) 247.

[10] Y. Aoki et al., Phys. Rev. D78 (2008) 054510.

[11] C. Sturm et al., Phys. Rev. D80 (2009) 014501.

[12] Y. Aoki, PoS LATTICE 2008 (2008) 222. 
[13] M. A. Donnellan et al., PoS LAT2007 (2007) 369.

[14] P. Nogueira, J. Comput. Phys. 105 (1993) 279.

[15] T. Seidensticker, Automatic application of successive asymptotic expansions of Feynman diagrams, hep-ph/9905298 (1999).

[16] T. Seidensticker, PhD thesis, Shaker, 2001, ISBN-13: 978-3826589430.

[17] R. Harlander, T. Seidensticker and M. Steinhauser, Phys. Lett. B426 (1998) 125.

[18] K. G. Chetyrkin and F. V. Tkachov, Nucl. Phys. B192 (1981) 159.

[19] S. Laporta and E. Remiddi, Phys. Lett. B379 (1996) 283.

[20] S. Laporta, Int. J. Mod. Phys. A15 (2000) 5087.

[21] J. A. M. Vermaseren, New features of FORM, math-ph/0010025 (2000).

[22] J. A. M. Vermaseren, Nucl. Phys. Proc. Suppl. 116 (2003) 343.

[23] M. Tentyukov and J. A. M. Vermaseren, Comput. Phys. Commun. 176 (2007) 385.

[24] R. H. Lewis, Fermat User Guide, http://www.bway.net/^lewis/.

[25] P. Breitenlohner and D. Maison, Commun. Math. Phys. 52 (1977) 11.

[26] T. L. Trueman, Phys. Lett. B88 (1979) 331.

[27] S. A. Larin, Phys. Lett. B303 (1993) 113.

[28] A. I. Davydychev, J. Phys. A25 (1992) 5587.

[29] S. Moch, P. Uwer and S. Weinzierl, J. Math. Phys. 43 (2002) 3363.

[30] S. Moch and P. Uwer, Comput. Phys. Commun. 174 (2006) 759.

[31] D. Maitre, Comput. Phys. Commun. 174 (2006) 222.

[32] D. Maitre, Extension of HPL to complex arguments, hep-ph/0703052 (2007).

[33] T. Huber and D. Maitre, Comput. Phys. Commun. 178 (2008) 755.

[34] A. I. Davydychev and M. Y. Kalmykov, Nucl. Phys. B699 (2004) 3.

[35] M. Y. Kalmykov, JHEP 04 (2006) 056.

[36] N. I. Usyukina and A. I. Davydychev, Phys. Lett. B332 (1994) 159.

[37] T. G. Birthwright, E. W. N. Glover and P. Marquard, JHEP 09 (2004) 042. 
[38] K. G. Chetyrkin, M. Faisst, C. Sturm and M. Tentyukov, Nucl. Phys. B742 (2006) 208.

[39] D. J. Broadhurst and A. G. Grozin, Phys. Rev. D52 (1995) 4082.

[40] J. A. Gracey, Phys. Lett. B488 (2000) 175.

[41] M. Gorbahn and S. Jäger, Precise MS-bar light-quark masses from lattice QCD in the RI/SMOM scheme, arXiv:1004.3997 [hep-ph] (2010).

[42] E. Remiddi and J. A. M. Vermaseren, Int. J. Mod. Phys. A15 (2000) 725.

[43] A. I. Davydychev and M. Y. Kalmykov, Nucl. Phys. B605 (2001) 266.

[44] M. Y. Kalmykov and O. Veretin, Phys. Lett. B483 (2000) 315.

[45] D. J. Gross and F. Wilczek, Phys. Rev. Lett. 30 (1973) 1343.

[46] H. D. Politzer, Phys. Rev. Lett. 30 (1973) 1346.

[47] G. 't Hooft, report at the Marseille Conference on Yang-Mills Fields (1972).

[48] W. E. Caswell, Phys. Rev. Lett. 33 (1974) 244.

[49] D. R. T. Jones, Nucl. Phys. B75 (1974) 531.

[50] E. Egorian and O. V. Tarasov, Teor. Mat. Fiz. 41 (1979) 26.

[51] O. V. Tarasov, A. A. Vladimirov and A. Y. Zharkov, Phys. Lett. B93 (1980) 429.

[52] S. A. Larin and J. A. M. Vermaseren, Phys. Lett. B303 (1993) 334.

[53] T. van Ritbergen, J. A. M. Vermaseren and S. A. Larin, Phys. Lett. B400 (1997) 379.

[54] M. Czakon, Nucl. Phys. B710 (2005) 485.

[55] R. Tarrach, Nucl. Phys. B183 (1981) 384.

[56] O. Tarasov, preprint JINR P2-82-900.

[57] K. G. Chetyrkin, Phys. Lett. B404 (1997) 161.

[58] J. A. M. Vermaseren, S. A. Larin and T. van Ritbergen, Phys. Lett. B405 (1997) 327. 